\title{
Coronal mass ejection-related particle acceleration regions during a simple eruptive event
}

\author{
Carolina Salas-Matamoros ${ }^{1,5}$, Karl-Ludwig Klein ${ }^{1,2}$, and Alexis P. Rouillard ${ }^{3,4}$ \\ ${ }^{1}$ LESIA-UMR 8109 - Observatoire de Paris, PSL Research. Univ., CNRS, Univ. P \& M Curie and Paris-Diderot, 92190 Meudon, \\ France \\ e-mail: carolina.salas@obspm.fr \\ 2 Station de radioastronomie - Observatoire de Paris, PSL Research Univ., CNRS, University Orléans, OSUC, 18330 Nançay, France \\ 3 Institut de Recherche en Astrophysique et Planétologie, Université de Toulouse (UPS), 31028 Toulouse Cedex 4, France \\ ${ }^{4}$ Centre National de la Recherche Scientifique, UMR 5277, 31300 Toulouse, France \\ 5 Space Research Center, University of Costa Rica, 2060 San Jose, Costa Rica
}

Received 20 December 2015 / Accepted 23 March 2016

\begin{abstract}
An intriguing feature of many solar energetic particle (SEP) events is the detection of particles over a very extended range of longitudes in the heliosphere. This may be due to peculiarities of the magnetic field in the corona, to a broad accelerator, to cross-field transport of the particles, or to a combination of these processes. The eruptive flare on 26 April 2008 provided an opportunity to study relevant processes under particularly favourable conditions since it occurred in a very quiet solar and interplanetary environment. This enabled us to investigate the physical link between a single well-identified coronal mass ejection (CME), electron acceleration as traced by radio emission, and the production of SEPs. We conduct a detailed analysis, which combines radio observations (Nançay Radio Heliograph and Nançay Decametre Array, Wind/Waves spectrograph) with remote-sensing observations of the corona in extreme ultraviolet (EUV) and white light, as well as in situ measurements of energetic particles near 1AU (SoHO and STEREO spacecraft). By combining images taken from multiple vantage points, we were able to derive the time-dependent evolution of the 3D pressure front that was developing around the erupting CME. Magnetic reconnection in the post-CME current sheet accelerated electrons, which remained confined in closed magnetic fields in the corona, while the acceleration of escaping particles can be attributed to the pressure front ahead of the expanding CME. The CME accelerated electrons remotely from the parent active region, owing to the interaction of its laterally expanding flank, which was traced by an EUV wave, with the ambient corona. SEPs detected at one STEREO spacecraft and SoHO were accelerated later, when the frontal shock of the CME intercepted the spacecraft-connected interplanetary magnetic field line. The injection regions into the heliosphere inferred from the radio and SEP observations are separated in longitude by about $140^{\circ}$. The observations for this event show that it is misleading to interpret multi-spacecraft SEP measurements in terms of one acceleration region in the corona. The different acceleration regions are linked to different vantage points in the interplanetary space.
\end{abstract}

Key words. acceleration of particles - Sun: coronal mass ejections (CMEs) - Sun: particle emission - Sun: radio radiation solar-terrestrial relations - Sun: flares

\section{Introduction}

A correct theory of the acceleration and subsequent transport of solar energetic particles (SEPs) in the heliosphere must explain the wide range of heliolongitudes over which a given SEP event can be detected in the inner heliosphere. While this fact was known before (Wibberenz \& Cane 2006), the comprehensive imaging and in situ measurements taken by the Solar Terrestrial Relations Observatory (STEREO) mission have demonstrated that the release of energetic particles over a very broad range of longitudes is neither an exceptional fact nor is it restricted to particularly strong events (Wiedenbeck et al. 2010; Dresing et al. 2012, 2014; Lario et al. 2013; Gómez-Herrero et al. 2015). Besides interplanetary transport across field lines (Dröge et al. 2014) or the expansion of open magnetic field lines in the corona (Klein et al. 2008), a spatially extended accelerator is often considered. For instance, the shock produced by the high-speed expansion of a fast coronal mass ejection (CME) can accelerate particles to high energies (Zank et al. 2000; Lee 2005; Afanasiev et al. 2015). The prime evidence of the existence of these shocks in the corona are type II radio bursts (Smerd et al. 1962; Nelson \& Melrose 1985; Mann et al. 1995; Nindos et al. 2008). Extreme ultraviolet (EUV) and white-light imaging can be used to track the effect of the strong pressure fronts, which disrupt the low (e.g., EUV waves) and upper corona during the formation and eruption of CMEs (see, for example, the recent review by Warmuth 2015). The CME shock is a convenient explanation of why SEPs are detected at spacecraft that are poorly connected with the solar active region where the activity originates (Torsti et al. 1999; Krucker et al. 1999; Rouillard et al. 2012; Park et al. 2015). When EUV waves alone are considered, however, the onset time of SEPs measured near 1AU cannot always be explained by the spatio-temporal evolution of the wave (Miteva et al. 2014).

In this work, we use non-thermal radio emissions as tracers of electron acceleration and transport during the eruption of a CME on 26 April 2008, during otherwise very quiet solar conditions in the deep solar minimum between cycles 23 and 24. This enables a study that does not suffer from coincidental associations of phenomena related with different events that happen at 
the same time. To map the plasma environment of the CME and its parent active region, we use EUV imaging and white-light coronagraphy from the STEREO and SoHO spacecraft, and advanced techniques of detailed modeling based on complementary sets of remote-sensing observations (Rouillard et al. 2016).

An overview of the event is given in Sect. 2, followed by a detailed description of the imaging and spectrographic observations of the radio emission (Sect. 3). The radio emission consisted of a broadband continuum from trapped electrons above the parent active region, and electron beams and a shock wave at remote locations. The connection between the regions is provided by an EUV wave. Its relation to the remote radio bursts is described in Sect. 4. SEPs were seen by one of the STEREO spacecraft and by SoHO (Sect. 5). The multi-spacecraft CME observations are modelled in Sect. 6, and used in a comparison with the radio imaging and spectrography to establish the relationship with the type III and type II bursts, and to derive parameters of the type II shock. A qualitative discussion of the most plausible mechanisms for particle acceleration during the development of this event is given in Sect. 7.

\section{Overview of the event on 26 April 2008}

On 26 April 2008, during the minimum of solar cycle 24, the Large Angle and Spectrometric Coronagraph experiment (LASCO; Brueckner et al., 1995) of the Solar and Heliospheric Observatory (SoHO) recorded a CME at 14:30 UT. This CME was associated with a weak B 3.8 soft X-ray (SXR) burst that occurred in an unnumbered spotless active region at $\mathrm{N} 10^{\circ} \mathrm{E} 10^{\circ}$. The STEREO spacecraft were located at $14^{\circ}$ east (STEREO B henceforth STB), and $35^{\circ}$ west (STEREO A - STA), from the active region. The EUV imagers on board the SoHO (Delaboudinière et al. 1995) and STEREO (Wuelser et al. 2004) spacecraft observed the initial development of the event. The CME appeared some time later as a halo CME in coronagraphic images from STB and as an east limb event in STA images. Using a reconstruction technique, Wood \& Howard (2009) interpreted the white-light images of the $\mathrm{CME}$ as a flux rope driving a bright shock.

The formation and evolution of the flux rope were studied by Huang et al. (2011), Temmer et al. (2011), and Cheng et al. (2012). Huang et al. (2011) argue that the formation of the flux rope could be traced by radio and EUV observations. The flux rope expanded and erupted leaving two footprints in the low corona imaged as two EUV dimmings on either side of the active region, where an arcade of loops formed. Temmer et al. (2011) describe in detail the propagation of the EUV waves away from the dimming.

The observations of the CME by the Sun-Earth Connection Coronal and Heliospheric Investigation (SECCHI) telescopes aboard STEREO permitted reconstructions of the CME structure and kinematics in and beyond the COR-2 field of view, above about $10 R_{\odot}$ (Wood \& Howard 2009; Thernisien et al. 2009; Temmer et al. 2011). From their 3D reconstruction, using the coronographs on board STEREO spacecraft, Wood \& Howard (2009) find an average CME velocity of $676 \mathrm{~km} \mathrm{~s}^{-1}$, while Thernisien et al. (2009) find $741 \mathrm{~km}^{-1}$. The studies cited above make no attempt to reconstruct the morphology of the solar eruptive event in the EUV field of view. Such a reconstruction is presented in this paper, in combination with the coronographs.
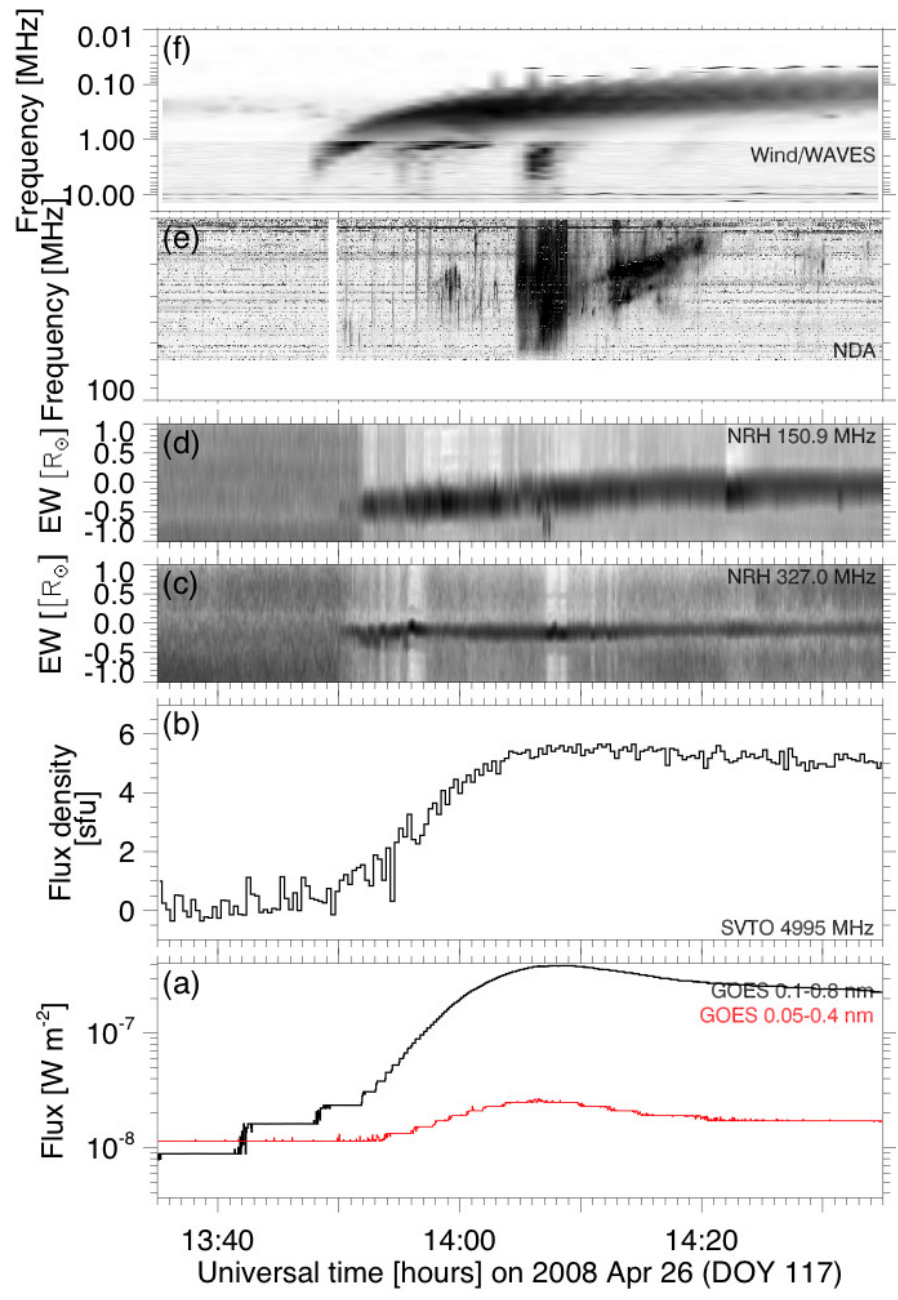

Fig. 1. Time series of the soft $X$-ray and radio emissions: a) soft $X$-ray profile; b) microwave profile at $5 \mathrm{GHz} ; \mathbf{c}), \mathbf{d}) 1 \mathrm{D}$ images projected onto the solar east-west direction at 150 and $327 \mathrm{MHz}$ ( $y$ axis graded in solar radii from the eastern to the western limb); e), f) dynamic spectra between $70 \mathrm{MHz}$ and $10 \mathrm{kHz}$

\section{Time evolution of the SXR and radio emission}

\subsection{Overview}

The multifrequency plot in Fig. 1 provides a complete visualization of the event. The soft X-ray burst observed by the Geosynchronous Operational Environmental Satellites (GOES; NOAA) in the bottom panel starts at 13:50 UT and peaks at 14:08 UT. The smooth microwave profile at $5 \mathrm{GHz}$ in Fig. $1 \mathrm{~b}$ is similar to the soft X-rays. The usual gyrosynchrotron emission that reveals mildly relativistic electrons in the low corona during flares is lacking, which suggests that there was no substantial electron acceleration to energies above $100 \mathrm{keV}$ in the flaring active region.

The two central panels, Figs. 1c and d, show the space-time imaging by the Nançay Radio Heliograph (NRH; Kerdraon \& Delouis 1997) at 150.9 and $327 \mathrm{MHz}$, respectively. 1D brightness scans as a function of the east-west position on the Sun are calculated from images integrated over $10 \mathrm{~s}$. The 1D scans are then scaled to show both weak and strong emissions. They are stacked and plotted as a grayscale image with time on the abscissa and the position on the solar east-west axis on the ordinate. The position is given in multiples of the solar radius, from the east limb $(-1)$ to the west limb $(+1)$. The gray surface before 13:50 UT shows the quiet corona. A broadband emission 
Universal time [hours] on 2008 Apr 26 (DOY 117)

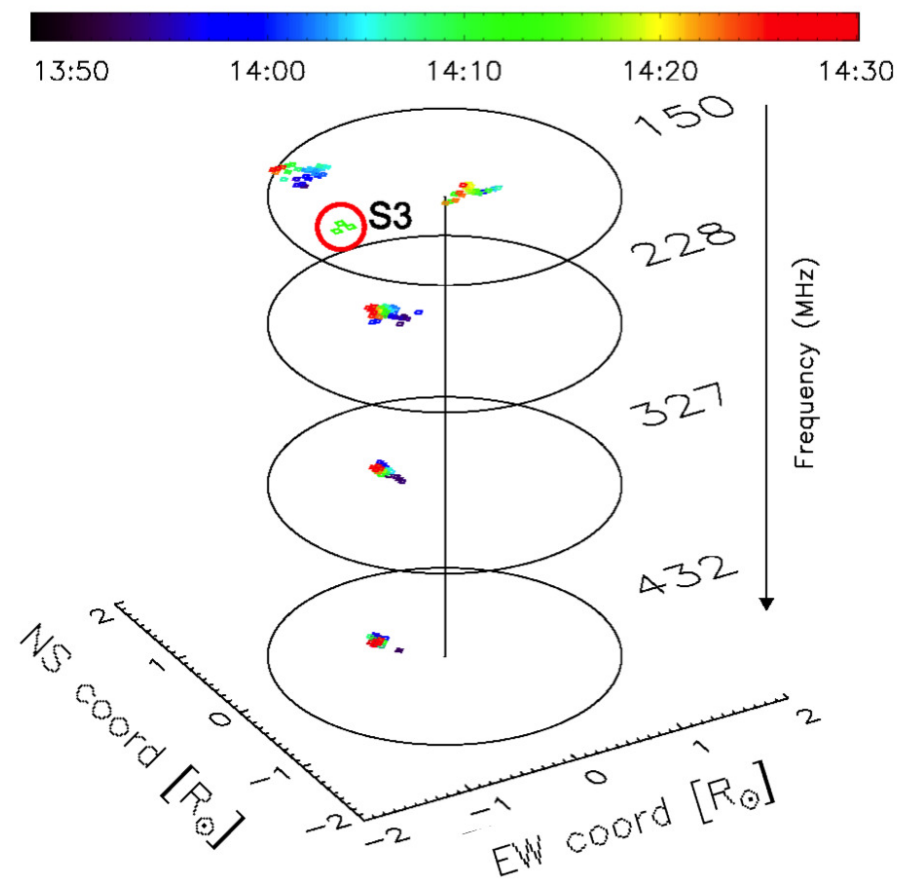

Fig. 2. 4D multifrequency plot showing the time evolution of the peak position in each image of the NRH at four frequencies. Time is represented by the color of the plot symbol, as indicated in the color bar at the top. The positions of the type III bursts (S3) near 14:07 UT at 150.9 MHz are enclosed in the red circle

(type IV burst) starts shortly after the SXR onset, and persists until the end of the observation (15:18 UT). This emission is detected at both frequencies. This source is located in the eastern hemisphere and presents an apparent movement westwards at $150.9 \mathrm{MHz}$ until around 14:30 UT when it becomes more stationary, with only a slight movement at $327 \mathrm{MHz}$. A different source, located to the east of the type IV burst, appears around 14:06:30 UT at 150.9 MHz.

Figure 2 shows how the position of the brightest pixel evolves in the course of time at four frequencies. The color of the plotted points denotes the time as indicated in the color bar at the top. The figure shows that the type IV source at the higher frequencies $(228,327,432 \mathrm{MHz})$ behaved differently from $150.9 \mathrm{MHz}$ : at the higher frequencies the source appeared simple, with a slight north-eastward motion during the burst. At $150.9 \mathrm{MHz}$, three distinct sources were seen: two appeared throughout the burst, both north-eastwards and south-westwards of the source at higher frequencies. As discussed by Huang et al. (2011), the type IV sources outline the expansion of a flux rope at $150.9 \mathrm{MHz}$ and emissions related to the current sheet below the flux rope at higher frequencies. Also, Fig. 2 shows the distinct locations of the bursts near 14:07 UT at $150.9 \mathrm{MHz}$ (S3, enclosed in the red circle). The green diamonds mark the positions of three successive peaks in NRH data using a cadence of $1 \mathrm{~s}$.

The dynamic spectrum observed by the Nançay Decametre Array (NDA; Lecacheux 2000) at long metre-waves (20-80 MHz) in Fig. 1e, shows few faint type III bursts that extend down to at least $20 \mathrm{MHz}$ (13:50-13:58 UT) followed by bursts that show a clear low-frequency cut-of, which was observed between 13:58 and 14:02 UT. The most prominent features in the spectrum are a group of broadband bursts between 14:04 and 14:09 UT and a type II burst, which starts during or immediately after this group and lasts until 14:21 UT. These two radio features will be discussed in the following sections. The WIND/Waves instrument (Bougeret et al. 1995) detects three interplanetary type III burst groups at low frequencies (see Fig. 1f). The first starts before 13:50 UT, which is before the type IV burst, at the start of the SXR and microwave bursts. Subsequently, two weak bursts accompany the faint type III bursts observed by NDA. A strong type III burst is associated with the bright broadband emission (14:04-14:09 UT) in the 20-80 MHz range. Because of the similar timing, we assume that these spectral features are the low-frequency counterpart of the radio source $\mathrm{S} 3$ detected far from the eruptive active region in the NRH images.

The type IV emission suggests that electrons are accelerated in the post-CME current sheet up to some $\mathrm{keV}$ or some tens of $\mathrm{keV}$. The exact energies of electrons producing the broadband plasma emission are not known. While some electrons escape to the high corona and the interplanetary space in the early phase of the type IV burst (faint type III bursts between 13:50 and 13:58 UT), others are injected into closed expanding magnetic structures, emitting the bursts with the drifting low-frequency cut-off between 13:58 and 14:02 UT. Unusual features are the broadband and type II bursts seen by NDA, because they occur late during the event, 15 min after the start of the type IV burst, and because the source of the broadband bursts seems to be far from the eruptive active region.

\subsection{Remote type III burst}

The differential spectrum of Fig. $3 c$ reveals that the bright 20-80 MHz emission between 14:04 and 14:09 UT is a group of type III bursts. We can identify individual bursts that start at about $70 \mathrm{MHz}$ and continue beyond $20 \mathrm{MHz}$. The burst group is time-related with the $150.9 \mathrm{MHz}$ source S3 in Fig. 2. On the low frequency side the source is accompanied by type III bursts observed by WIND/Waves (Figs. 1e and f).

The spinning of the Wind spacecraft can, in principle, be used to infer the direction of radio emission arrival (Reiner 2001). This direction finding technique can be applied in the low-frequency channel RAD1, at frequencies below 1.075 MHz. Until 14:00 UT, the direction finding observations (courtesy S.Hoang) show an azimuth at $1.04 \mathrm{MHz}$ slightly east of central meridian (fluctuating around $2^{\circ}$ ) and an elevation north of the ecliptic, with a broad scatter around an average of $4^{\circ}$. The type III burst, observed between about 14:06 and 14:12, is clearly seen above the decaying flux density at $1.04 \mathrm{MHz}$, but with less and less contrast as the frequency decreases. At $548 \mathrm{kHz}$ it is hidden in the decaying flux of the previous bursts. This burst is the low-frequency counterpart of the strong type III group seen by NDA and of the burst at $150.9 \mathrm{MHz}$ that was observed by NRH (S3, Fig. 2). At the time of this burst, the direction finding at $1.04 \mathrm{MHz}$ shows a peak at about $6^{\circ}$ azimuth, east of the previous type III bursts. As expected, no distinct position can be identified at lower frequencies, given the low contrast of the burst.

The direction finding observations by Wind/Waves are consistent with the location S3 of the bursts at $150.9 \mathrm{MHz}$ : both show radio emissions from sources that are well to the east of the earlier emission, which is related to the flaring active region. The temporal association with the type III bursts observed by NDA thus suggests that the $150 \mathrm{MHz}$ emission of source $\mathrm{S} 3$ is also produced by electron beams which propagate outward, or else by downward-propagating electron beams. A third possibility are metric spikes, which are often found near 

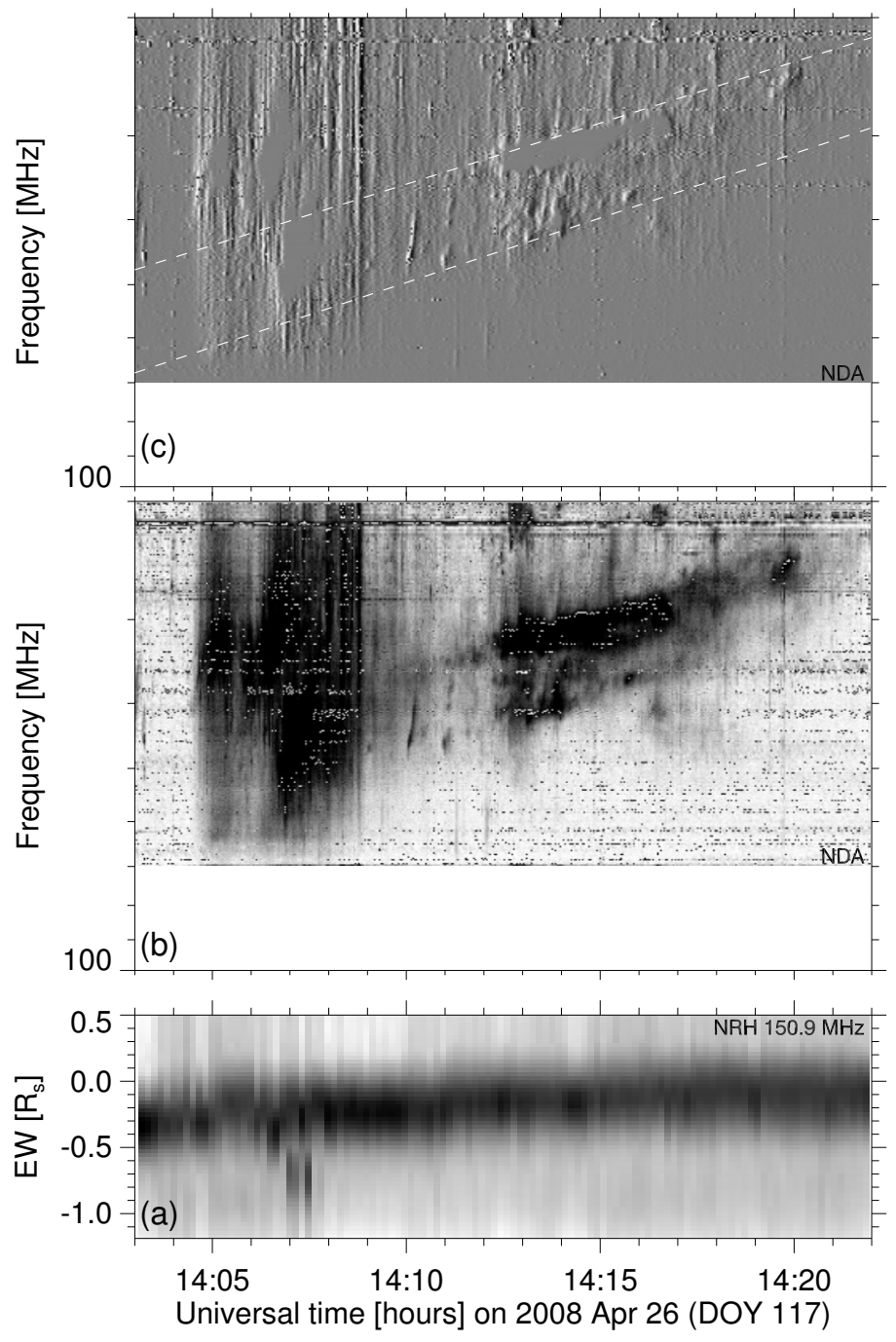

Fig. 3. Time history of the metre-wave radio emission during the late type III and type II bursts: a) 1D images projected onto the solar eastwest direction at $150 \mathrm{MHz}$ ( $y$-axis graded in solar radii from the eastern to the western limb) showing a continuum emission and a sporadic source on its eastern side at around 14:07 UT, b) dynamic spectrum between 70 and $20 \mathrm{MHz}$ and c) time-difference spectrum of b). The dashed lines are fits of the upper and lower borders of the type II burst in b).

the starting frequencies of type III bursts (Paesold et al. 2001). We cannot differentiate between these possibilities, because we have no detailed spectrum around $150 \mathrm{MHz}$. But in all cases the acceleration region in the corona is near S3. The relation between this electron acceleration and the eruptive activity will be discussed in the following sections.

\subsection{Type II burst}

The type II burst (14:10-14:21 UT) follows the group of type III bursts. The dynamic spectrum in Fig. 3b shows a regular drift, which suggests that the exciter travels along a smooth density gradient. The exciter of a type II burst is considered to be a region of a shock wave where electrons that are able to produce radio emission via plasma instabilities are accelerated (Holman \& Pesses 1983). We determined the drift rate of the low-frequency $\left(v_{\mathrm{LF}}\right)$ and high-frequency $\left(v_{\mathrm{HF}}\right)$ borders by identifying ten points in each band with the cursor between 14:10 and 14:19 UT, and fit straight lines in the time$\log$ (frequency) plane, minimizing the absolute deviation. The drift rate of the low-frequency band of the type II burst is $\frac{\mathrm{d}}{\mathrm{d} t} \ln v=-7.0 \times 10^{-4} \mathrm{~s}^{-1}$, and of the high-frequency band $-7.4 \times 10^{-4} \mathrm{~s}^{-1}$. The uncertainty of the drift rate is about $10 \%$, if we assume an uncertainty of $5 \%$ of the cut-off frequencies inferred visually from the dynamic spectrogram. The dashed white lines overplotted on the differential dynamic spectrum in Fig. 3c are the fits in the time- $\log$ (frequency) plane.

To transform the frequency drift into the propagation speed of the exciter, we consider an isothermal hydrostatic density distribution with scale height $H\left(r_{o}\right)$ developed around the height $r_{o}$, which corresponds to the central frequency of the type II burst, $v_{o}=30 \mathrm{MHz}$. If the exciter travels at speed $V_{\mathrm{II}}$ along a direction that is inclined to the radial direction by an angle $\theta$, the drift of the logarithm of the frequency $v$ is

$\frac{\mathrm{d}}{\mathrm{d} t} \ln v=-\frac{V_{\mathrm{II}} \cos \theta}{2 H\left(r_{o}\right)}$.

In the isothermal hydrostatic model,

$H\left(r_{o}\right)=\left(\frac{r_{o}}{R_{\odot}}\right)^{2} H\left(R_{\odot}\right)=50 \times 10^{6}\left(\frac{r_{o}}{R_{\odot}}\right)^{2} \frac{T}{1 \mathrm{MK}}[\mathrm{m}]$.

So, for a temperature $T=1.5 \mathrm{MK}$ the radial speed of the exciter at the height $r_{o}$ inferred from the drift of the low-frequency type II band is

$V_{\mathrm{rad}}=V_{\mathrm{II}} \cdot \cos \theta=105 \cdot\left(\frac{r_{o}}{R_{\odot}}\right)^{2}\left[\mathrm{~km} \mathrm{~s}^{-1}\right]$.

The uncertainties from the fit of the type II drift rate formally carry over to an uncertainty of about $10 \%$ in all speed estimates. Using the fitted values of the upper and lower frequency limits of the type II burst, we find that the frequency ratio is on average 1.39 , and that the relative bandwidth is on average 0.32 with a statistical uncertainty of \pm 0.01 of both values.

\section{Magnetic configuration and EUV-wave observations}

The PFSS extrapolation of Schrijver \& De Rosa (2003) based on SoHO/MDI magnetic field measurements of the entire solar disk shows closed magnetic structures in a wide region around the flaring active region. Only the open field lines are plotted in Fig. 4a. The type III burst sources S3 at $150.9 \mathrm{MHz}$ were found in the red square. They project onto open magnetic field lines at the south-eastern border of the large region with closed fields around the flare site. These open field lines correspond to a narrow coronal hole seen in EUV images (SoHO/EIT) and in NRH Earth-rotation synthesis images (C. Mercier, priv. comm.).

From the 3D coordinates of the open PFSS magnetic field lines through the square, the radial distance of the type III burst source at $150.9 \mathrm{MHz}$ is found to be $1.7 R_{\odot}$. This heliocentric distance is very high compared to usual estimates of type III burst source heights at $150 \mathrm{MHz}$ (Saint-Hilaire et al. 2013), and appears especially high in the present event where the magnetic field configuration is far from the active region. A possible explanation is that the open magnetic field lines are affected by the impinging CME, as will be discussed below. The above value should not be considered as the real altitude of the source S3.

The two open PFSS lines through the type III source plotted in Fig. $4 \mathrm{~b}$ are connected to the source surface at longitudes E69 


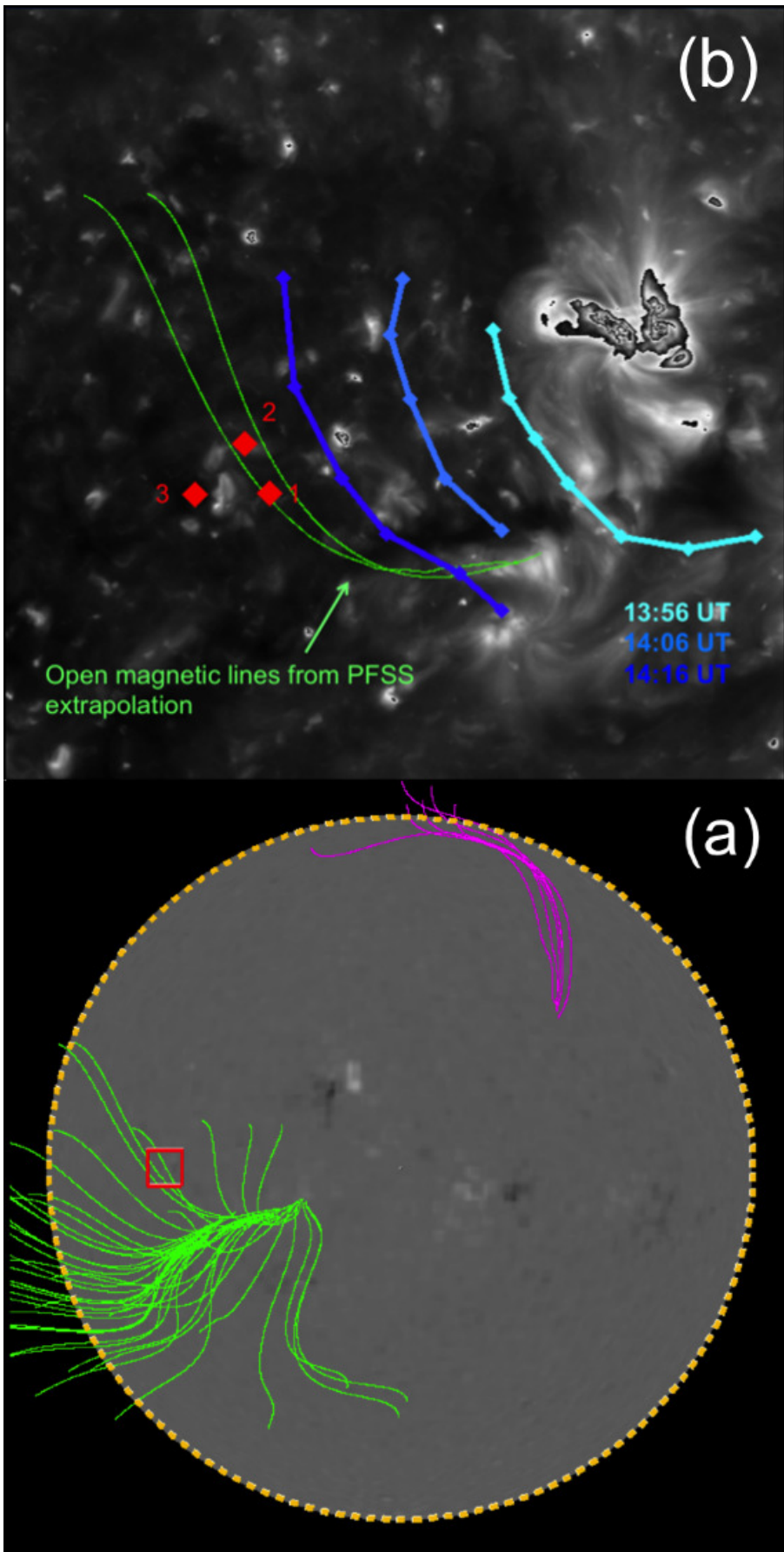

Fig. 4. a): Source region of the type III bursts (red square) over the open magnetic field lines (in green) inferred using the PFSS extrapolation model by Schrijver \& De Rosa (2003). b): Superposition of two open magnetic field lines (in green) close to the positions of three successive individual type III bursts (red diamonds) on the $19.5 \mathrm{~nm}$ EUVI synoptic map from STEREO B. The blue lines outline the EUVI wave front at different times: 13:56, 14:06 (near the time of the type III burst), and 14:16 UT.

and $E 77^{\circ}$. The interplanetary Parker spirals rooted there are plotted as dashed curves in Fig. 5. They were computed using a solar angular speed of $1.664 \times 10^{-4} \circ \mathrm{s}^{-1}$ (rotation period 25.3 days) and an average solar wind speed of $420 \mathrm{~km} \mathrm{~s}^{-1}$. The direction to the interplanetary type III burst measured by WIND/Waves is plotted by the solid line in Fig. 5. This line crosses the Parker spiral field lines at a heliocentric distance of around $19 R_{\odot}$. The comparison with the locations of the STEREO, SoHO, and Wind

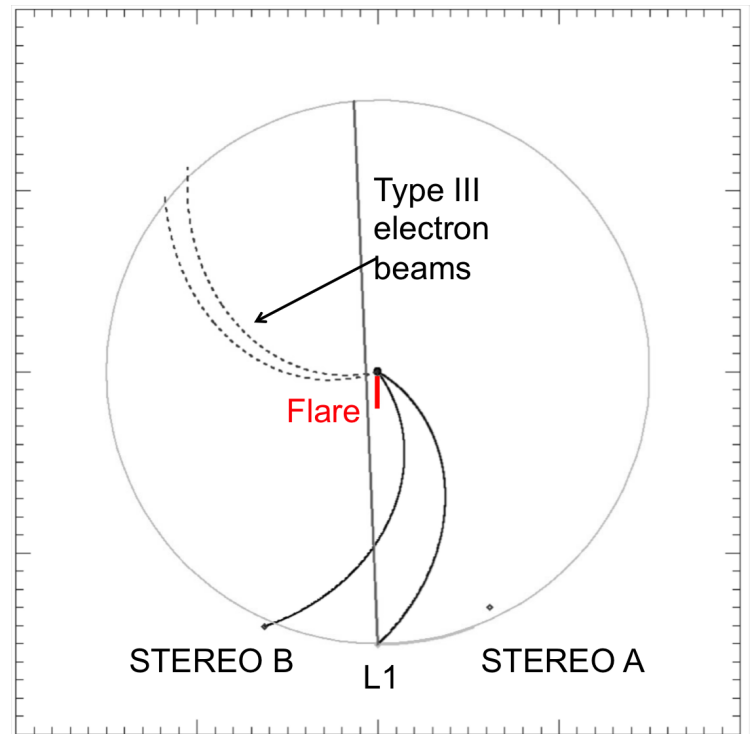

Fig. 5. Schematic configuration of the three spacecraft positions: L1 (SoHO and WIND), STEREO A, and STEREO B. The curved lines correspond to the Parker spiral connecting the source surface to STEREO B and to Earth. The dashed lines represent the Parker spirals connected at the source surface $\left(2.5 R_{\odot}\right)$ to the open magnetic field lines through the type III source at $150 \mathrm{MHz}$. The solid line shows the line of sight from the Wind/Waves experiment to the type III burst source at $1.04 \mathrm{MHz}$.

spacecraft in Fig. 5 shows that the electron beams accelerated near source S3 are released onto interplanetary field lines, which are not connected to any spacecraft.

The acceleration of these electrons far from the active region requires an alternative accelerator to the flare process. An EUV wave observed by STEREO/EUVI is a possible candidate. The lateral expansion of the EUV wave was tracked. Its front is traced at three different times by light and dark blue lines in Fig. 4b. The propagation speed along the solar surface is found to be $207 \mathrm{~km} \mathrm{~s}^{-1}$. The central (medium blue) line shows the wave front as measured at 14:06 UT. This observation implies that the CME flank reaches the footpoints of the open magnetic field lines through the radio source S3 near the time when the type III bursts appear. This is evidence that the interaction of the EUV wave with the open magnetic field lines triggered the acceleration of the electron beams that caused the type III bursts far from the flaring active region.

\section{Solar energetic particles}

The Solar Electron and Proton Telescope (SEPT; Müller-Mellin et al. 2008) aboard STB detected a tiny electron event in close time relationship with the eruption. Figure $6 b$ shows that the intensity of electrons streaming away from the Sun starts to rise near 14:00 UT, peaks around 16:00 UT, and then decays until 18:00 UT, when a new rise starts. Even though this weak peak is time-related with the eruption, a similar peak was observed near 06:00 UT without an associated flare. This casts doubt on the association of the enhancement between 14 and $16 \mathrm{UT}$ with the eruptive flare.

The radio observations suggest that some electrons might escape from the type IV source region. This is consistent with the weak electron event. The electrons from S3 have no magnetic connection through the nominal Parker spiral with STB (Fig. 5). An association between the electron intensity enhancement seen at STB with this type III burst is hence not plausible. 

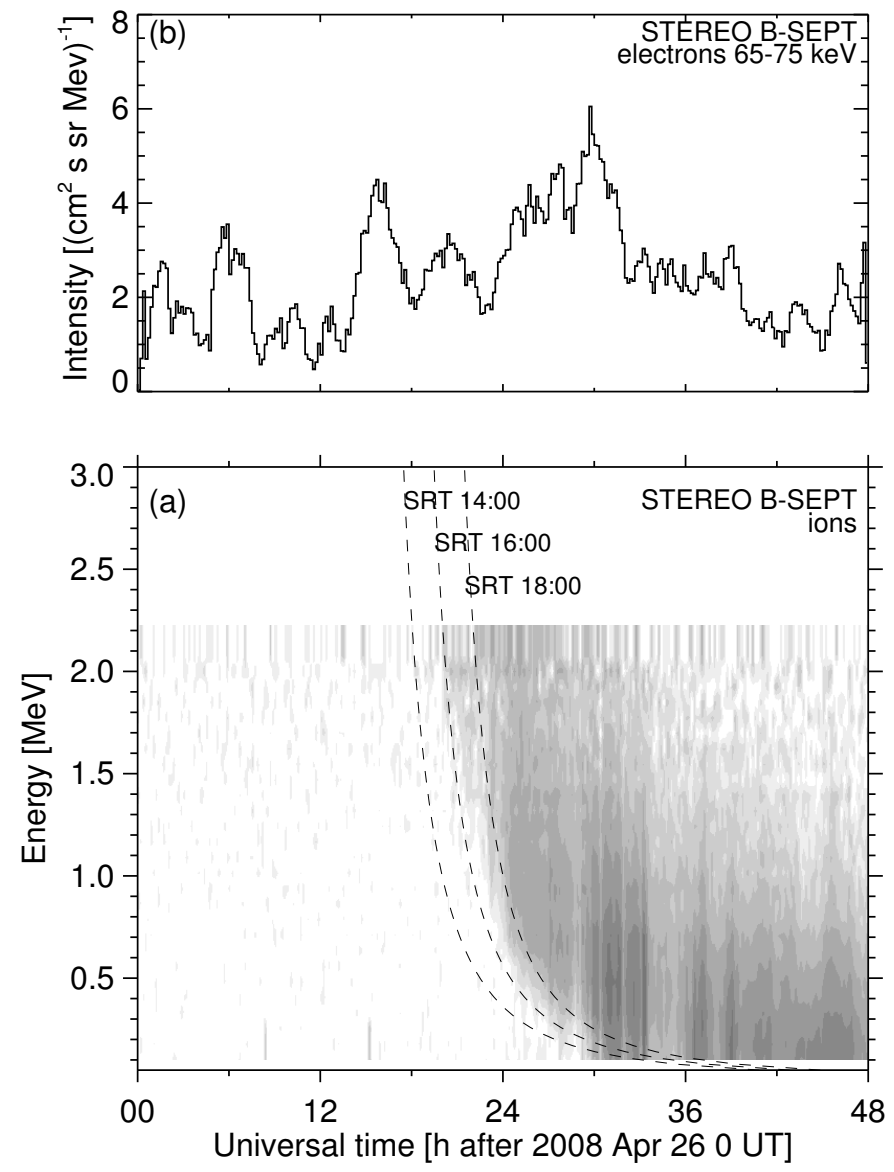

Fig. 6. Time evolution of solar energetic particle (SEP) intensities as seen by the sunward looking detectors of STEREO B/SEPT. a) Dynamic spectrum representing the 30 channels of the SEPT instrument. The dashed curves give the expected arrival time of protons at the spacecraft for three different solar release times (SRT) assuming an interplanetary path length of $2 \mathrm{AU}$. b) Electron intensity.

The electron enhancement could also be associated with the type II burst, but we argue below that the type II source is at a similar location as $\mathrm{S} 3$.

The STB/SEPT also observed an ion event. Figure 6a shows, in the form of a dynamical spectrum, the ion intensities normalized to their pre-event background. The ions detected by SEPT are considered to be mostly protons unless the proton spectrum is steep (Müller-Mellin et al. 2008), and protons are expected to arrive at the spacecraft before the heavy ions. Therefore, in the following, we consider that the first ions arriving at the detector are protons. A weaker, but significant SEP event was also observed in the (4-7) MeV channels of the Electron Proton Helium Instrument (EPHIN, Müller-Mellin et al. 1995) aboard SoHO.

The three dashed curves in Fig. 6a show the expected arrival times of protons at STB as a function of their energy, for three different solar release times. We assume that the protons travel a path of $2 \mathrm{AU}$. This is longer than the Parker spiral, but may account for a prolonged travel path owing to particle scattering by the turbulent interplanetary magnetic field (see Laitinen et al. 2015, and references therein). These curves suggest that a proton injection starting near 14:00 UT is not consistent with the data. A more plausible solar release time is 16:00 UT, but this value is of course only a rough estimate. The SXR and microwave profiles do not show any other burst on 26 April. Neither do the observations of WIND/Waves present any interplanetary type III burst that would reveal fresh particle injections after

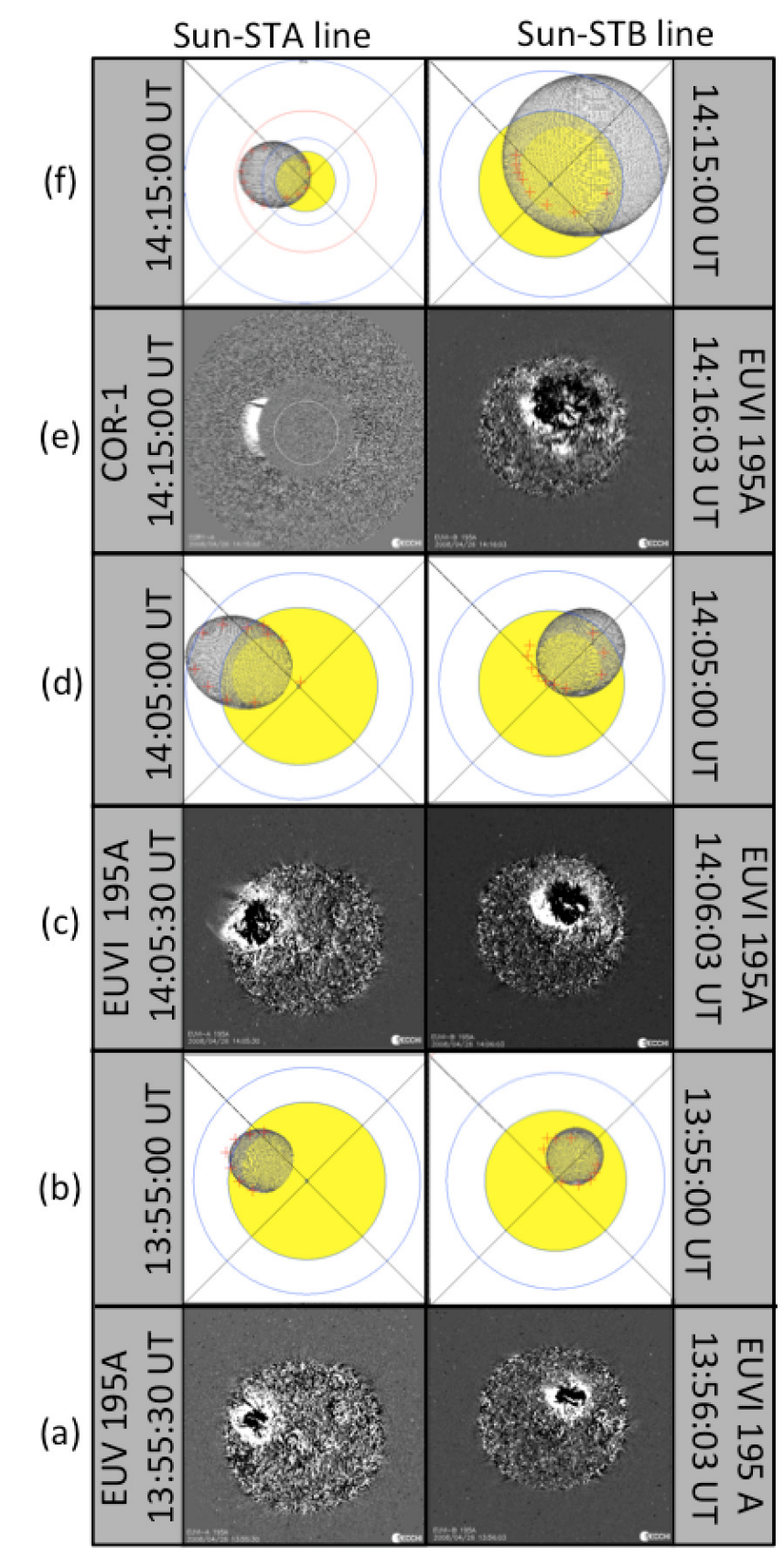

Fig. 7. Comparison of running-difference images (rows a), c), e)) of the eruptive event observed by STA (left hand-column) and STB (righthand column) with the results of applying the fitting technique (rows b), d), f)) developed by Rouillard et al. (2016). The images are all from the EUVI instruments, except the left-hand image shown in row f) obtained by COR1-A. Red crosses superposed on the fitted ellipsoids show the contour of the propagating front observed in the running difference images and are used to constrain the extent and location of the ellipsoid at each time.

14:10 UT. Therefore, the most plausible candidate for the ion acceleration and later electron acceleration is the CME high in the corona. The height-time trajectory of the CME front seen by STA, shown in Fig. 7 of Cheng et al. (2010) and also in Fig. 6 of Temmer et al. (2011), suggests a distance from the solar surface of $7 R_{\odot}$ at 16:00 UT.

\section{Comparison with 3D CME modeling}

Rouillard et al. (2016) present a new technique to derive the properties of the $3 \mathrm{D}$ expansion of pressure fronts forming in the 

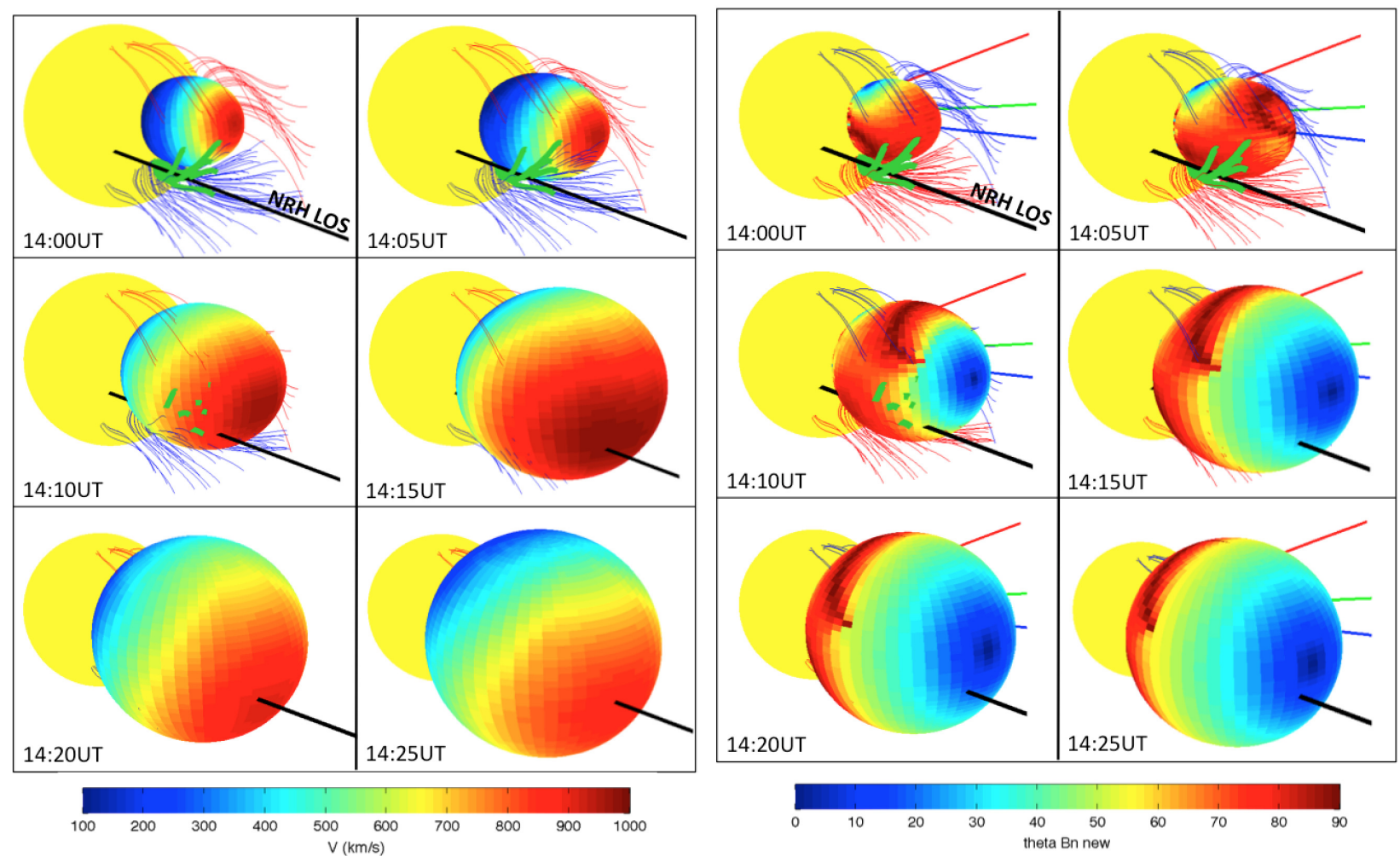

Fig. 8. 3D evolution of the CME at different times from 14:00 UT to 14:25 UT. The color code shows the distribution of the speed (left panel) and the angle between the normal on the front and the direction of the upstream magnetic field lines (right panel). The open magnetic field lines are plotted in red (positive polarity) and blue (negative polarity). The thick black line is the line of sight (LOS) from Earth to the type III source at $150 \mathrm{MHz}$. The green lines are the open field lines intercepted by the LOS.

corona during eruptive events. The technique uses a combination of EUV and white-light images and maps of the outermost extent of the coronal region perturbed by the CME as a function of time. In this respect, the technique is similar to the technique proposed by Lario et al. (2014). Rows a, c, and e of Fig. 7 present images covering the first $20 \mathrm{~min}$ of the CME eruption as viewed along the Sun-STA and Sun-STB lines. The surface of the pressure front generated around the expanding $\mathrm{CME}$ is visible in these EUV and white-light images, it is initially fairly regular and we found that an ellipsoid fits the outermost extent of this perturbed region very well. We manually extracted the location of the outermost extent of the pressure front formed around the CME for all cameras and at all available times. These points are plotted as red crosses in the images given in rows b, $\mathrm{d}$, and $\mathrm{f}$, and are used to outline the contour of the ellipsoids fitted in this study.

Just as for other CME events, we found that the ellipsoid that passes through the contour of the pressure front observed in coronagraphic images intersects the solar surface at the location of the EUV wave. The EUV wave is here considered the low-coronal counterpart of the expanding front surrounding the erupting flux rope.

Our technique goes beyond previous studies (e.g., Kwon et al. 2014) in the following manner: once the parameters of the successive ellipsoids are obtained, we interpolate these parameters at regular time steps of $\delta t=300 \mathrm{~s}$ to generate a sequence of regularly time-spaced ellipsoids. To compute the 3D expansion speed of the surface of the pressure wave, we find for a point $P$ on the ellipsoid at time $t+\delta t$, the location of the closest point on the ellipsoid at previous time-step $t$. We then compute the distance travelled between these two points, which we divide by the time interval $\delta t=300 \mathrm{~s}$ to obtain an estimate of the speed of the disturbance at point $P$.

The left panel in Fig. 8 presents the results of extracting the normal speed of the pressure front at these six successive times displayed as a color-coded speed distribution over the front surface. In addition, we show the location of open magnetic field lines derived using the same PFSS model as in Fig. 4. In this computation, the source surface was set at $2.5 R_{\odot}$.

Only open field lines derived from the PFSS extrapolation are shown in Fig. 8. They trace the location of the streamer out of which the CME emerges, with (in red/blue) inward/outward pointing field lines. Also shown, in green, are the open field lines that are located along the line of sight of the type III burst imaged by the NRH instrument. The line of sight from the Earth to the source S3 of type III bursts is shown as a black line labeled "NRH LOS" in these figures.

\subsection{CME and type III bursts}

It had been shown in Sect. 4 that the type III bursts occur far from the flaring active region, when the EUV wave impacts onto the open magnetic field lines shown by the PFSS extrapolation (see Fig. 4).

The triangulation at 14:00 UT in Fig. 8 shows that no magnetic field line open to the interplanetary medium is connected with the pressure front. At this time the CME presents an elongated shape, with a speed of just under $1000 \mathrm{~km} \mathrm{~s}^{-1}$ near its so-called nose. The speed decreases along the flank towards $200 \mathrm{~km} \mathrm{~s}^{-1}$ in the low corona near the location of the EUV wave. The CME is hence expected to drive a shock wave at and around its nose, but not at the lower parts of its flanks. At 14:05, near 
the onset of the type III bursts in the NDA spectrum, and two minutes before the start at $150 \mathrm{MHz}$, the pressure front is just about to pass through the open field lines situated near the southeastern flank. This confirms the idea that the type III bursts occur at the interface between the expanding CME and the open field lines of the coronal hole. At 14:10 UT, the speed of the front intersecting the green lines is greater than $700 \mathrm{~km} \mathrm{~s}^{-1}$ and the front has by then almost certainly steepened into a shock.

\subsection{CME and type II burst}

The type II burst starts at or immediately after the time of the type III emission, and is also observed much later than usual during the event. We therefore assume that the type III bursts and the type II burst are physically related, and the type II burst is another consequence of the interaction of the south-eastern CME flank with the open coronal magnetic field lines. Since the type II burst starts at lower frequency than the type III bursts, this interaction most likely takes place at a greater altitude than the type III source at $150 \mathrm{MHz}$. This is also consistent with the modeling result that the CME expansion is probably too slow to drive a shock wave at the low coronal altitude where the type III bursts originate, whereas speeds able to drive a shock are found at greater height (Fig. 8). The six right-hand panels in Fig. 8 present, in a similar format to the left-hand panels, the angle between the normal vector of the front and the direction of the ambient magnetic field lines derived from the PFSS model. Both open and closed magnetic field lines are considered for this derivation. The angle is close to 90 degrees, shown by red colors, over a large part of the south-eastern flank of the front at 14:10 UT. The quasi-perpendicular region rapidly shrinks, however, to the lower parts of the front as it proceeds to higher altitudes. We conclude that at 14:10 UT the shock surface is mostly quasi-perpendicular on the south-eastern flank of the CME.

Assuming the type II source is related to a shock wave at the south-eastern CME flank, we compared the height profiles of the radial component of its outward speed derived from the dynamic spectrum (Eq. (3)), plotted by the solid line in Fig. 9a, and of the radial component of the expansion speed on the south-eastern CME flank inferred from the 3D modeling, plotted by filled triangles and fitted by a parabola, as shown by the dashed curve. The gray band represents the $\pm 10 \%$ uncertainty of the type II speed discussed above. The two curves intersect at heliocentric distance $r_{o}=2.1 \pm 0.3 R_{\odot}$. We recall that $r_{0}$ denotes the height where the central frequency of the type II burst $(30 \mathrm{MHz})$ is emitted. At this distance, the angle between the CME surface and the radial direction, whose height profile is plotted in Fig. $9 \mathrm{~b}$, is $54^{\circ}$ with a range of uncertainty $\left(42^{\circ}-63^{\circ}\right)$ induced by the uncertainty of the height where the two parabolas intersect in Fig. 9a. These quantities are listed in Table 1, together with the parameters derived from the dynamic spectrum of the type II burst.

Table 2 summarizes the model-dependent derivation of further parameters of the shock wave. We do not include error estimates, since a considerable uncertainty comes from various model assumptions, such as a hydrostatic isothermal electron density at a temperature of $1.5 \mathrm{MK}$. The quantitative indications are meant to give an idea of how consistent the results are with respect to other work. Their relevance to coronal physics has to be discussed in the framework of the model assumptions. The start height of the type II source (line 1) is deduced from the hydrostatic density model. Since the low-frequency side of the type II spectrum is generally assumed to come from the upstream plasma, which has not yet been disturbed by the CME, the hydrostatic assumption is not unreasonable. The upstream
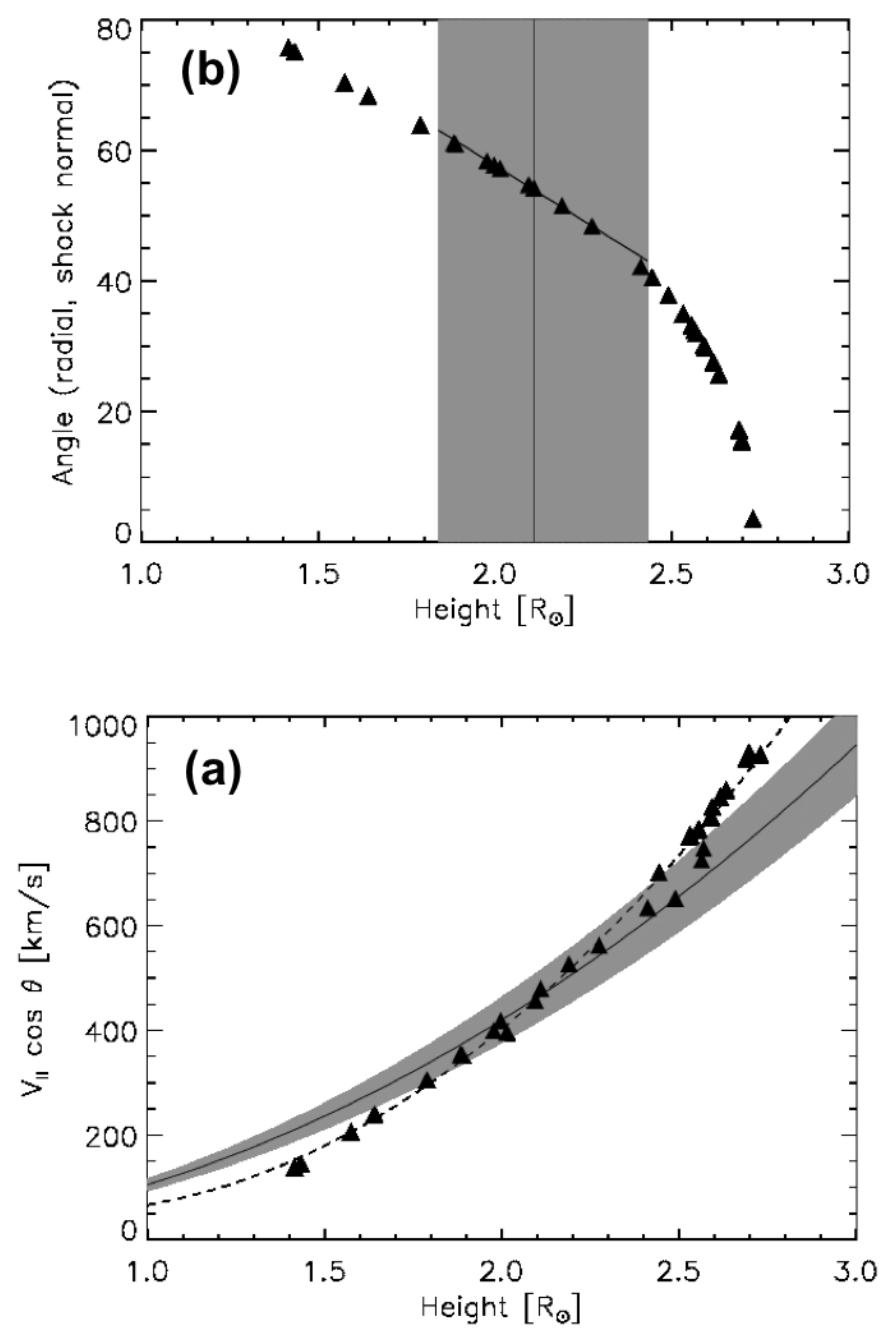

Fig. 9. a) Radial speed of the CME and the type II exciter. Solid line: type II exciter from Eq. (3). Symbols: radial component of the expansion CME speed on the south-eastern flank. The dashed line: quadratic fit of these points. The gray band shows the $\pm 10 \%$ uncertainty of the type II exciter speed. b) Height profile of the angle between the normal to the CME front and the radial direction. Symbols: angles inferred from the CME modeling. Inclined solid line: linear fit of the points around the reference height $r_{o}$. Vertical solid line: corresponding angle at the reference height $r_{o}=2.1 R_{\odot}$. The gray band presents the uncertainty of the height owing to the type II exciter speed.

Table 1. Parameters of type II burst.

\begin{tabular}{lr}
\hline \hline Type II burst spectrum & \\
\hline Centre frequency $v_{0}$ & $30 \mathrm{MHz}$ \\
Start frequency $v_{\mathrm{st}}$ & $40 \mathrm{MHz}$ \\
High-to-low frequency ratio & 1.39 \\
Frequency drift rate $(\log )$ & $(-7.0 \pm 0.7) \times 10^{-4} \mathrm{~s}^{-1}$ \\
\hline CME modeling & \\
\hline Central height $r_{0}$ & $(2.1 \pm 0.3) R_{\odot}$ \\
Angle $(\boldsymbol{n}$, radial $)$ & $54^{\circ}\left(42^{\circ}-63^{\circ}\right)$ \\
\hline
\end{tabular}

electron density and mass density (lines 4 and 5) are directly determined by the low-frequency limit of the type II burst. The values given in the table refer to the emission frequency of $v_{0}=30 \mathrm{MHz}$, hence to a plasma frequency of $15 \mathrm{MHz}$ in the 
Table 2. Inferred parameters of the type II shock.

\begin{tabular}{lrr}
\hline \hline (1) Start height & $r_{\mathrm{st}}=r_{0}\left(1+\frac{2 H\left(r_{0}\right)}{r_{0}} \ln \frac{v_{\mathrm{st}}}{v_{0}}\right)^{-1}$ & $1.9 R_{\odot}$ \\
(2) Height extent & $\Delta r \leq 2 H\left(r_{0}\right) \ln \frac{\nu_{\mathrm{HF}}}{v_{\mathrm{LF}}}$ & $\leq 0.32 R_{\odot}$ \\
(3) Exciter speed $V_{\mathrm{II}}$ & $V_{\mathrm{II}}=-\frac{2 H\left(r_{0}\right)}{\cos \theta} \frac{\mathrm{d}}{\mathrm{d} t} \ln v$ & $800 \mathrm{~km} \mathrm{~s}^{-1}$ \\
(4) Upstream electron density $\left(r_{0}\right)$ & $n_{\mathrm{eu}}=\frac{4 \pi^{2} \epsilon_{0} m_{\mathrm{e}}}{e^{2}}\left(\frac{v_{0}}{2}\right)^{2}$ & $2.8 \times 10^{12} \mathrm{~m}^{-3}$ \\
(5) Upstream mass density $\left(r_{0}\right)$ & $\rho_{\mathrm{u}}=1.14 n_{\mathrm{eu}} m_{\mathrm{p}}$ & $5.3 \times 10^{-15} \mathrm{~kg} \mathrm{~m}^{-3}$ \\
(6) Density compression & $X=\left(\frac{v_{\mathrm{HF}}}{v_{\mathrm{LF}}}\right)^{2}$ & 1.9 \\
(7) Alfvén Mach number & $M_{\mathrm{A}}=\sqrt{\frac{X}{2} \frac{5+5 \beta+X}{4-X}}$ & \\
(8) Upstream Alfvén speed $\left(r_{0}\right)$ & $c_{\mathrm{Au}}=\frac{V_{\mathrm{II}}}{M_{\mathrm{A}}}$ & \\
(9) Upstream magnetic field $\left(r_{0}\right)$ & $B_{\mathrm{Au}}=\sqrt{\mu_{0} \rho_{\mathrm{u}}} c_{\mathrm{Au}}$ & \\
(10) Upstream plasma $\beta\left(r_{0}\right)$ & $\frac{2}{\gamma}\left(\frac{c_{\mathrm{s}}}{c_{\mathrm{Au}}}\right)^{2}$ & $0.33 \mathrm{~km} \mathrm{~s}$ \\
\hline
\end{tabular}

usual assumption that when a single type II band is seen, the emission is harmonic.

The instantaneous width of the type II burst spectrum can be used to estimate the height extent in the same hydrostatic model (line 2). This is an upper limit, where we assume that the entire type II burst emission comes from the upstream region. The estimate suggests that the type II source only occupies a fraction of the surface of the CME. This is consistent with the general assumption that type II emission comes from the quasiperpendicular region of a shock. The right panel of Fig. 8 shows that in the snapshots during the type II burst (14:10-14:20 UT) the quasi-perpendicular region, shown by the red color, covers a substantial, but decreasing, part of the CME flank. It is tempting to relate the well-defined end of the type II burst near 14:21 UT to the development of an increasingly large quasi-parallel geometry on the CME front in regions of high expansion speed. At 14:25 UT the quasi-perpendicular part of the CME front is exclusively at low altitudes, where the expansion speed is probably too low to drive a shock wave. The exciter speed of the type II shock follows from Eq. (3) and the parameters in Table 1.

If we adopt the interpretation of band splitting in type II bursts as revealing simultaneous emission from the upstream and downstream plasma (Smerd et al. 1974; Vršnak et al. 2001), the frequency ratio of the split bands gives the density compression ratio at the shock (Table 2, line 6). This determines the Alfvénic Mach number (line 7), where the polytropic index $\gamma$ and the $\beta$ of the plasma are undetermined. We assume $\gamma=5 / 3$ and leave $\beta$ as an unknown. Using the exciter speed $V_{\mathrm{II}}$, we can then calculate the Alfvén speed and the magnetic field strength upstream of the shock, still with an unknown $\beta$. The relationship between the plasma beta and the Alfvén and sound speeds is given in the last line of the table. Since we know the sound speed, we can insert the upstream Alfvén speed into this expression, with $\beta$ as an unknown, then determine $\beta$, and use its value to quantify the Alfvénic Mach number, the Alfvén speed, and the magnetic field strength. All these parameters refer to the height $r_{o}=2.1 \pm 0.3 R_{\odot}$.

\subsection{CME shock and SEP acceleration}

Particles accelerated in the quasi perpendicular shock (revealed by the type II burst) will not be observed by any spacecraft because of their location in the interplanetary space (Fig. 5). These particles reach $1 \mathrm{AU}$ at a heliolongitude of around $-137^{\circ}$ relative to L1 and the Earth. All spacecraft are magnetically connected to the western regions of the flare-CME system.

We applied the same triangulation technique as used in Fig. 8 to locate the extent of the pressure front much higher in the solar atmosphere when the CME has sufficiently expanded to intersect the nominal Parker spiral connected with STB. The triangulation work was carried out by considering both the COR-2 coronagraph, as well as the inner heliospheric imager (HI-1).

To infer how STB connects with the low corona at the time of the event, we must verify that the solar wind situated between the corona and STB is not significantly disturbed. To do so, we considered the STEREO catalogs of CMEs and corotating interaction regions (CIRs), which were made available by the Heliospheric Cataloguing, Analysis and Techniques Service (HELCATS) FP7 project. Since STA was directly imaging the Sun-STB line (see Fig. 5), the catalogs are perfectly suited for this analysis. The analysis reveals that (1) the CME of interest to the present paper was the only one observed by STEREO for many days before and after the event; (2) a CIR was also passing in the field of view of $\mathrm{HI}$ at the same time and should have hit STB at roughly the same time as the CME shock. Comparison of the location of both structures during their propagation to 1 AU shows that the magnetic connectivity of STB to the western part of the shock (Fig. 5) could not have been altered by the formation of the CIR on 26 April 2008. The CIR was forming well upstream of the CME but was not present near the heights 


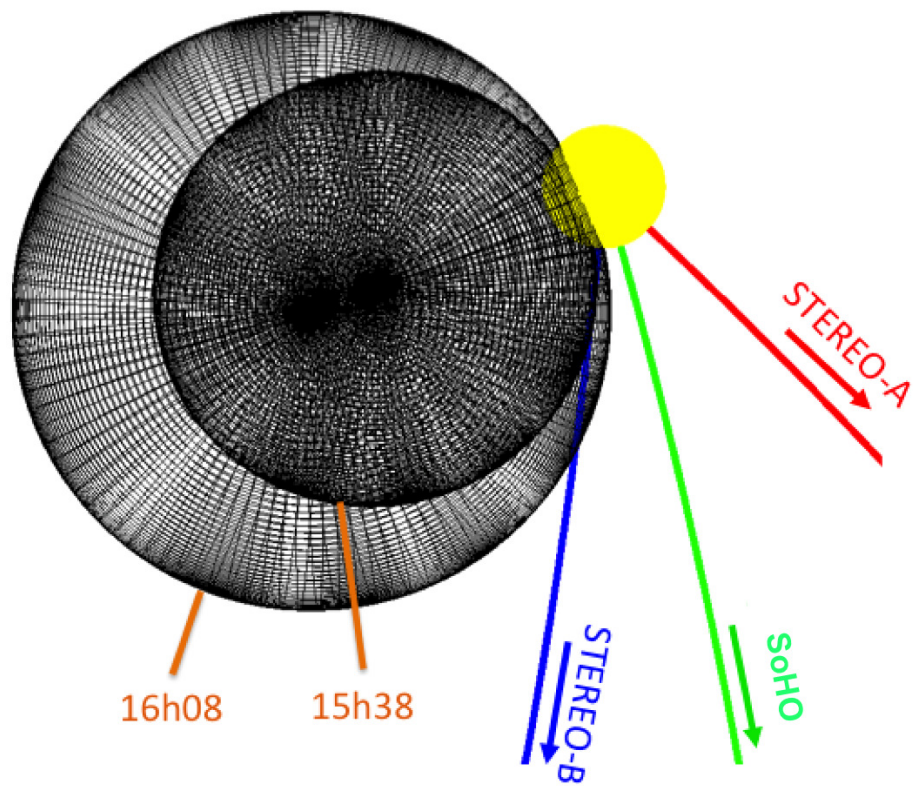

Fig. 10. Pressure wave triangulated from STEREO/COR2 observations at 15:38 UT and at 16:08 UT, as seen by an observer above the northern solar pole. The three labeled lines represent the Parker spiral field lines in the ecliptic plane that connect the parent active region to the spacecraft.

and longitudes of the shock at 15:38 UT and at 16:08 UT on 26 April 2008. For more details, see the analysis presented in Appendix A.

The triangulation work carried out high up in the corona using COR-2 and HI-1 confirms the gradual southward and eastward shift of the central axis of the pressure front that was already detected in EUVI and COR-1 (Sect. 6). The longitudinal shift goes from $216^{\circ}$ at $13: 55 \mathrm{UT}$ to $200^{\circ}$ at 16:08 UT.

The derived position of the propagating front at these later times reveals that the western CME flank intercepts the interplanetary field lines connected with the STB spacecraft after 15:38 UT (Fig. 10). This is consistent with the injection time, which was estimated by considering the measured ion time profile discussed in Sect. 5 (Fig. 6). A similar configuration between the CME shock and the Parker spiral has been analysed and modelled in Rouillard et al. (2011). Careful analysis of the magnetic connectivity of the shock with the particle detectors also showed a delayed onset of the SEP event (about $16 \mathrm{~h}$ ), which was attributed to the time for the modelled shock to intersect the relevant magnetic field lines.

\section{Discussion}

The eruptive event on 26 April 2008 illustrates different energetic particle populations associated with different acceleration sites related to a CME. The observations are summarized as follows:

1. The event showed no evidence of electron acceleration to mildly relativistic energies in the low corona: the microwave emission had a purely thermal character.

2. Electron acceleration was first observed above the flaring active region, most likely related to the post-eruptive current sheet, through the type IV continuum seen over a broad range of dm-to-m-waves. Spectrography from longer metre-wavelengths to kilometre-wavelengths suggests that few electrons could escape. This is consistent with the largescale closed magnetic configuration revealed by the PFSS extrapolation.

3. Type III bursts from electron beams and a type II burst from a coronal shock wave were observed unusually late, more than $15 \mathrm{~min}$ after the start of the eruptive event. The type III source could be imaged at $150 \mathrm{MHz}$, and was found to be located far south-eastward of the flaring active region, on open field lines revealed by the PFSS extrapolation and indirectly by a coronal hole in EUV and radio images. An EUV wave was found to arrive at the open field lines related to the type III bursts near the time of the bursts. The 3D modeling of the stereoscopic CME observations confirms the occurrence of the type III bursts, and therefore the acceleration of the electron beams, as the south-eastern flank of the laterally expanding CME impacted the open magnetic field lines of the coronal hole. At 1 AU the Parker spirals connected to those PFSS field lines which traverse the type III burst source are separated by $\sim 110^{\circ}$ in heliolongitude from the nearest spacecraft, STB.

4. Parameters of the shock wave were derived from the combination of the spectrographic observations and the CME modeling under the assumption that the type II burst also occurred on the south-eastern flank of the CME. This leads to a fairly complete, but model-dependent, description of the shock and the upstream plasma. The geometry of the CME flank at the presumed site of the type II burst is quasi-perpendicular.

5. The shock at the front of the CME is not observed directly, but its presence is strongly suggested by the high speed $\sim 1000 \mathrm{~km} \mathrm{~s}^{-1}$. Its geometry is quasi-perpendicular during the first 5-10 min of the CME rise. Thereafter it becomes quasi-parallel, and the quasi-parallel geometry occupies an increasing fraction of the CME surface as the event progresses.

6. SEPs up to several MeV are observed by the best-connected spacecraft, STB. Their intensity starts to rise nearly two hours after the start of the eruption. The Parker spiral through the spacecraft originates westward of the eruptive active region. The expanding CME reaches this field line near the time when the SEPs seen at the spacecraft were released at the Sun.

7. The radio observations of electron acceleration at the Sun and of SEPs near 1 AU reveal particle acceleration at different regions of an expanding CME. They cover a remarkably extended range of heliolongitudes of about $140^{\circ}$.

The high cadence of the data and the multiple view points during this event give us a unique opportunity to see different particle populations from different acceleration sites linked to the same CME. The observations allow us to discuss qualitatively different acceleration mechanisms involved.

\subsection{Type III burst and particle acceleration during the interaction of the CME with the coronal hole}

STEREO and SoHO images have shown an EUV wave reaching a coronal hole close to the type III burst source position at $150 \mathrm{MHz}$ (S3). The observed speed of the EUV wave, $207 \mathrm{~km} \mathrm{~s}^{-1}$, and the expansion of the CME front inferred from stereoscopic modeling at heliocentric distances of $<1.5 R_{\odot}$, are unlikely to be fast enough to reveal a shock. The shock traced by the type II burst likely occurred at a greater height than S3. 
Thus, we can conclude that even though both sources, S3 and the type II burst, are most likely located in the coronal hole region and are linked to the expansion of the CME, they are independent manifestations of the CME impact on the coronal hole. Therefore, an alternative accelerator is needed to explain the electron acceleration near S3 taking into account the interaction of the EUV wave with its surroundings.

In solar plasmas, the magnetic reconnection process is normally related to the energy release in flares that can accelerate energetic particles (e.g., Aschwanden 2002). Nevertheless, the magnetic reconnection can also happen when the expanding magnetic structure of the CME that formed in the active region interacts with the surrounding field lines (Dasso et al. 2006). Magnetic reconnection can inject energetic electrons onto open magnetic field lines in two ways.

On the one hand, if the CME magnetic structure is filled with energetic electrons, the reconnection with the surroundings will give them access to open magnetic field lines. This was envisaged by Schatten \& Mullan (1977) and modelled by Masson et al. (2013). Evidence of electrons confined in the flux rope was presented in Sect. 3. However, radio emission from these accelerated electrons was observed only north-eastward and south-westward of the active region (see Fig. 2), far from region S3. So the scenario is not supported by the observations, although we cannot exclude it definitely, because we do not fully understand the radiation process.

An alternative idea is that the particles are locally accelerated near the S3 location. In a magnetic reconnection scenario related to flares, pairs of oppositely drifting bursts (type III and reverse slope) are expected. These pairs of bursts that start simultaneously and at the same frequency reveal electrons that are accelerated upwards and downwards from the reconnection region (Aschwanden 2002). These pairs of bursts are not observed in the spectrum in Fig. 3, although we cannot exclude the idea that the bursts at $150 \mathrm{MHz}$ are reverse slope bursts. In addition, downward-propagating electron beams may not be observed because the plasma is compressed, so that the enhanced collision rate or turbulence is able to isotropize the beams, while the enhanced magnetic field is able to reflect them.

Another process that must be considered because of the magnetic compression is betatron acceleration. Since the magnetic moment is conserved in a collisionless plasma, the particles gain perpendicular kinetic energy when the local magnetic field increases (e.g., Baumjohann \& Treumann 1996). The increase in kinetic energy is equal to that of the magnetic field. If the plasma is slightly collisional or subjected to wave turbulence, the perpendicular momentum can be transferred to parallel momentum.

We examine if this process can be effective in accelerating the electrons through the compression of the open magnetic field in the coronal hole by the impact of the CME. If we use the reference height of the type II burst $\left(r_{o}=2.1 R_{\odot}\right.$, Sect. 6), we find that the height of the S3 source at $150 \mathrm{MHz}$ is $r=1.2 R_{\odot}$. At this altitude the magnetic field given by the PFSS extrapolation is $B \simeq 0.49 \mathrm{G}$. To estimate the magnetic field compression, we compare this value with an upper limit reached during the compression, namely the magnetic field required to stop the CME expansion by the build-up of magnetic pressure at the interface of the CME and the coronal hole. The compressed magnetic field $(B)$ was calculated from the condition of equilibrium between the dynamic pressure of the expanding CME and the magnetic pressure in the compressed open magnetic flux tube:

$B=\sqrt{2 \cdot \mu_{0} \rho} \cdot V \simeq 1.47[\mathrm{G}]$, with $\rho=1.14 n_{\mathrm{e}} m_{\mathrm{p}}$. The electronic density inside the CME $\left(n_{e}\right)$ was taken $12 \%$ higher than the ambient density (Kozarev et al. 2011; Schrijver et al. 2011). The ambient electron density was found to be $6.9 \times 10^{7} \mathrm{~cm}^{-3}$, assuming harmonic emission of the source at $150.9 \mathrm{MHz}$. The speed of the impact, $V$, is the velocity of the EUV wave.

Considering that electrons at speeds around three times the thermal speed in the ambient plasma can be accelerated by the magnetic field compression, we obtain an energy of about $3.5 \mathrm{keV}$. This is less than the energy usually associated with type III emitting electron beams in the corona (Alvarez \& Haddock 1973; Lin 1974; Poquerusse 1994; Klassen et al. 2003). In the present case, we can estimate the exciter speed of the type III burst from the drift rate. In fact no drift is discernible in the type III bursts of the differential spectrum in Fig. 3 between 70 and $20 \mathrm{MHz}$. Given that the integration time is $2.5 \mathrm{~s}$, this implies a lower limit of the absolute value of the logarithmic drift rate of $0.5 \mathrm{~s}^{-1}$ and a lower limit of the exciter speed of $0.4 c$, which corresponds to a kinetic energy of about $45 \mathrm{keV}$. This value is higher than the one estimated from the compression ratio, which was already a generous estimate of an upper limit. Successive episodes of magnetic pumping might be more efficient, for instance if the CME expansion produced large-amplitude fast magnetosonic waves.

In conclusion, both magnetic reconnection and betatron acceleration can qualitatively account for the acceleration of type III emitting electron beams, but we have no definite observational evidence to distinguish between them.

\section{2. $C M E$ shock}

Because of its high speed, the CME is expected to drive a shock wave. This is consistent with the occurrence of a type II radio burst. Although only spectral observations of this burst were available, the combination with the CME modeling gave valuable, albeit model-dependent, insights into the type II burst and its role in the particle acceleration at the CME shock. The physical relationship between the metre-wave type II burst and the CME is strongly supported by the timing and the coincidence with the impact of the south-eastern flank of the CME on the coronal hole.

\subsubsection{CME shock and type II radio burst}

The emission of metre-wave type II bursts on the flank of a CME was reported in a number of recent studies (e.g., Magdalenić et al. 2014; Zucca et al. 2014a), although evidence on a location near the nose is also frequent, especially at altitudes within a solar radius above the photosphere (Dauphin et al. 2006; Zimovets et al. 2012; Zucca et al. 2014b). In the present case, no imaging observations of the type II burst are available, but the consistency between the type II spectrum and the height profile of the expansion velocity derived from the stereoscopic CME modeling strongly argues for a source location on the flank. Using this constraint, we infer that the shock geometry must be quasi-perpendicular. This again adds evidence to existing knowledge (Steinolfson 1984; Zimovets et al. 2012), using a new technique. The observations suggest that the type II emission ceases as the quasi-perpendicular part of the CME front shrinks to a small region of relatively low expansion speed in the low corona. This is a new possible interpretation of the finite duration of metre-wave type II bursts. 
The Mach number of the type II shock, as inferred from the hypothesis of simultaneous emission upstream and downstream of the shock front, is moderate, $M_{A}=1.9$. The value is consistent with previous work (Vršnak et al. 2002; Mancuso \& Garzelli 2013; Kouloumvakos et al. 2014; Vasanth et al. 2014; Zucca et al. 2014b), but it is more closely constrained in the present event by the combination of the type II spectrum and the stereoscopic CME modeling. Similar Mach numbers were derived from white-light observations (Bemporad \& Mancuso 2010, 2011).

The interpretation of band splitting in terms of simultaneous emission upstream and downstream of the shock was challenged on theoretical grounds (Cairns 2011), and alternative ideas were developed (McLean 1967; Treumann \& LaBelle 1992; Sakai \& Karlický 2008). Those which localize the split-band sources in different regions upstream of the shock front have not been confirmed by imaging observations. Zimovets et al. (2012) and Zucca et al. (2014b) present two case studies where multifrequency imaging show the high-frequency split band to be slightly, but systematically, displaced inward with respect to the low-frequency split band, which is in agreement with the hypothesis of simultaneous emissions from the upstream and downstream region. Another major support to this interpretation is the finding that, in type II bursts where the feature was observed in interplanetary space, the in situ density measurements upstream and downstream of the shock wave near 1 AU were indeed consistent with the Earthward extrapolation of the type II split bands (Vršnak et al. 2001). Finally, numerical simulations also show that shock-accelerated electrons may penetrate into the downstream region (Savoini et al. 2005).

\subsubsection{CME shock and SEP acceleration}

CMEs are thought to accelerate particles over an extended spatial range. The Mach number found on the south-eastern flank of the 26 April 2008 CME corresponds to a subcritical shock in quasiperpendicular geometry (see Fig. 5 of Mann et al. 1995). It is therefore not clear if this part of the CME shock was able to accelerate protons. Electrons that were accelerated at this shock would be expected to be seen about $120^{\circ}$ eastward of the flaring active region.

The first protons observed in interplanetary space were accelerated when the part of the shock that was situated close to the western flank of the Sun was magnetically connected to the spacecraft. In the present event, this occurred only when the CME front was far from the Sun. The SEPs detected at STEREO B and SoHO complete the manifestations of particle acceleration at this particular CME, demonstrating that physically different accelerators are at work in different acceleration regions, so that the remotely observed particle signatures depend on the region of the CME front to which the observer is connected.

\section{Summary and conclusions}

The occurrence of an eruptive event on 26 April 2008 during very quiet coronal conditions gave us the opportunity to identify different energetic particle populations originating in different acceleration sites that were triggered by the evolution of the CME. In summary, we were able to determine the relationship between the CME expansion, the EUV wave, and the particle acceleration regions:

1. No non-thermal electrons are seen from the flaring active region itself. The acceleration occurs only higher in the corona as was revealed by decimetric and decametric radio emission.

2. Energetic electrons were accelerated, which produced type III burst emission at the interaction region between the south-eastern CME flank and the ambient corona, far from the active region. Candidate acceleration processes operating there are magnetic reconnection and compressional acceleration at the interface between the CME flank and the corona.

3. The shock revealed by the type II burst was most likely also located at the south-eastern CME flank, but at a slightly greater height $\left(\sim 1.9 R_{\odot}\right)$. The local geometry was found to be quasi-perpendicular. While the shock wave was clearly able to accelerate electrons, its inferred Mach number suggests that it was sub-critical and therefore not an efficient proton accelerator.

4. The late SEP event (MeV protons) observed at STEREO B and $\mathrm{SoHO}$ is associated with the shock-acceleration near the western CME flank, where the geometry was quasi-parallel. The late onset is consistent with the time when the pressure wave (CME border) higher in the corona became magnetically connected to the spacecraft.

5. The various acceleration regions identified during this event released electrons and/or protons over an extended range of heliolongitudes reaching nearly $140^{\circ}$.

The observations of this well-defined CME, which occurred in a rather simple environment that is typical of solar minimum, reveal the simultaneous or successive action of different acceleration regions. These acceleration regions are linked to different vantage points in the interplanetary space. While this example does show that a CME releases energetic particles into a broad range of heliolongitudes, it does demonstrate that multispacecraft SEP measurements may not probe one acceleration region in the corona.

Acknowledgements. The authors are indebted to Sang Hoang for providing the direction-finding analysis of the Wind/Waves observations. They acknowledge helpful discussions within the team The Connection Between Coronal Shock Wave Dynamics and Early SEP Production led by K. Kozarev and N. Nitta at the International Space Science Institute (ISSI) in Bern. C.S.-M. gratefully acknowledges the financial support of her doctorate studies by the University of Costa Rica and the Ministry of Science, Technology and Telecommunications of Costa Rica (MICITT) through the National Council of Scientific and Technological Research (CONICIT). This research was also supported by the Agence Nationale pour la Recherche (ANR/ASTRID, DGA) project Outils radioastronomiques pour la météorologie de l'espace (ORME, contract No. ANR-14-ASTR-0027) and by the French space agency (CNES). A.P.R. acknowledges use of the tools made available by the French plasma physics data centre (Centre de Données de la Physique des Plasmas; CDPP; http://cdpp.eu/), CNES and the space weather team in Toulouse (Solar-Terrestrial Observations and Modelling Service; STORMS). This includes the data-mining tools AMDA (http: //amda.cdpp.eu/), the CLWEB tool (clweb.cesr.fr/) and the propagation tool (http://propagationtool.cdpp.eu). The catalogs used in the Appendix to track the location of the CME and CIRs in the interplanetary medium were created by the HELCATS project under the FP7 EU contract number 606692 .

\section{References}

Afanasiev, A., Battarbee, M., \& Vainio, R. 2015, A\&A, 584, A81

Alvarez, H., \& Haddock, F. T. 1973, Sol. Phys., 29, 197

Aschwanden, M. J. 2002, Space Sci. Rev., 101, 1

Baumjohann, W., \& Treumann, R. A. 1996, Basic space plasma physics (London: Imperial College Press)

Bemporad, A., \& Mancuso, S. 2010, ApJ, 720, 130

Bemporad, A., \& Mancuso, S. 2011, ApJ, 739, L64

Bougeret, J.-L., Kaiser, M. L., Kellogg, P. J., et al. 1995, Space Sci. Rev., 71, 231

Brueckner, G. E., Howard, R. A., Koomen, M. J., et al. 1995, Sol. Phys., 162, 357 
Cairns, I. H. 2011, in IAGA Special Sopron Book Series, Vol. 4, The Sun, the Solar Wind, and the Heliosphere, eds. M. P. Miralles, \& J. Sánchez Almeida (Springer), 267

Cheng, X., Ding, M. D., \& Zhang, J. 2010, ApJ, 712, 1302

Cheng, X., Zhang, J., Saar, S. H., \& Ding, M. D. 2012, ApJ, 761, 62

Dasso, S., Mandrini, C. H., Démoulin, P., \& Luoni, M. L. 2006, A\&A, 455, 349

Dauphin, C., Vilmer, N., \& Krucker, S. 2006, A\&A, 455, 339

Delaboudinière, J.-P., Artzner, G. E., Brunaud, J., et al. 1995, Sol. Phys., 162, 291

Dresing, N., Gómez-Herrero, R., Klassen, A., et al. 2012, Sol. Phys., 281, 281

Dresing, N., Gómez-Herrero, R., Heber, B., et al. 2014, A\&A, 567, A27

Dröge, W., Kartavykh, Y. Y., Dresing, N., Heber, B., \& Klassen, A. 2014, J. Geophys. Res. (Space Phys.), 119, 6074

Gómez-Herrero, R., Dresing, N., Klassen, A., et al. 2015, ApJ, 799, 55

Holman, G. D., \& Pesses, M. E. 1983, ApJ, 267, 837

Huang, J., Démoulin, P., Pick, M., et al. 2011, ApJ, 729, 107

Kerdraon, A., \& Delouis, J.-M. 1997, in Coronal Physics from Radio and Space Observations, ed. G. Trottet (Berlin: Springer Verlag), Lect. Notes Phys., 483, 192

Klassen, A., Karlický, M., \& Mann, G. 2003, A\&A, 410, 307

Klein, K.-L., Krucker, S., Lointier, G., \& Kerdraon, A. 2008, A\&A, 486, 589

Kouloumvakos, A., Patsourakos, S., Hillaris, A., et al. 2014, Sol. Phys., 289 2123

Kozarev, K. A., Korreck, K. E., Lobzin, V. V., Weber, M. A., \& Schwadron, N. A. 2011, ApJ, 733, L25

Krucker, S., Larson, D. E., Lin, R. P., \& Thompson, B. J. 1999, ApJ, 519, 864

Kwon, R.-Y., Zhang, J., \& Olmedo, O. 2014, ApJ, 794, 148

Laitinen, T., Huttunen-Heikinmaa, K., Valtonen, E., \& Dalla, S. 2015, ApJ, 806, 114

Lario, D., Aran, A., Gómez-Herrero, R., et al. 2013, ApJ, 767, 41

Lario, D., Raouafi, N. E., Kwon, R.-Y., et al. 2014, ApJ, 797, 8

Lecacheux, A. 2000, in Radio Astronomy at Long Wavelengths, eds. R. G. Stone K. W. Weiler, M. L. Goldstein, \& J.-L. Bougeret, AGU Geophys. Monogr. Ser., 119, 321

Lee, M. A. 2005, ApJS, 158, 38

Lin, R. P. 1974, Space Sci. Rev., 16, 189

Magdalenić, J., Marqué, C., Krupar, V., et al. 2014, ApJ, 791, 115

Mancuso, S., \& Garzelli, M. V. 2013, A\&A, 560, L1

Mann, G., Classen, T., \& Aurass, H. 1995, A\&A, 295, 775

Masson, S., Antiochos, S. K., \& DeVore, C. R. 2013, ApJ, 771, 82

McLean, D. J. 1967, PASA, 1, 47

Miteva, R., Klein, K.-L., Kienreich, I., et al. 2014, Sol. Phys., 289, 2601

Müller-Mellin, R., Kunow, H., Fleissner, V., et al. 1995, Sol. Phys., 162, 483

Müller-Mellin, R., Böttcher, S., Falenski, J., et al. 2008, Space Sci. Rev., 136, 363

Nelson, G. J., \& Melrose, D. B. 1985, in Solar Radiophysics: Studies of Emission from the Sun at Metre Wavelengths, eds. D. McLean, \& N. Labrum (Cambridge, Great Britain: Cambridge University Press), 333
Nindos, A., Aurass, H., Klein, K.-L., \& Trottet, G. 2008, Sol. Phys., 253, 3 Paesold, G., Benz, A. O., Klein, K.-L., \& Vilmer, N. 2001, A\&A, 371, 333 Park, J., Innes, D. E., Bucik, R., Moon, Y.-J., \& Kahler, S. W. 2015, ApJ, 808, 3 Plotnikov, I., Rouillard, A. P., Davies, J. A., et al. 2016, Sol. Phys., submitted Poquerusse, M. 1994, A\&A, 286, 611

Reiner, M. J. 2001, Space Sci. Rev., 97, 126

Rouillard, A. P., Davies, J. A., Lavraud, B., et al. 2010a, J. Geophys. Res. (Space Phys.), 115, A04103

Rouillard, A. P., Lavraud, B., Davies, J. A., et al. 2010b, J. Geophys. Res. (Space Phys.), 115, A04104

Rouillard, A. P., Odstrčil, D., Sheeley, N. R., et al. 2011, ApJ, 735, 7

Rouillard, A. P., Sheeley, N. R., Tylka, A., et al. 2012, ApJ, 752, 44

Rouillard, A. P., Plotnikov, I., Pinto, R., et al. 2016, ApJ, submitted

Saint-Hilaire, P., Vilmer, N., \& Kerdraon, A. 2013, ApJ, 762, 60

Sakai, J. I., \& Karlický, M. 2008, A\&A, 478, L15

Savoini, P., Lembège, B., Krasnosselskhik, V., \& Kuramitsu, Y. 2005, Ann. Geophys., 23, 3685

Schatten, K. H., \& Mullan, D. J. 1977, J. Geophys. Res., 82, 5609

Schrijver, C. J., \& De Rosa, M. L. 2003, Sol. Phys., 212, 165

Schrijver, C. J., Aulanier, G., Title, A. M., Pariat, E., \& Delannée, C. 2011, ApJ, 738,167

Smerd, S. F., Wild, J. P., \& Sheridan, K. V. 1962, Austr. J. Phys., 15, 180

Smerd, S. F., Sheridan, K. V., \& Stewart, R. T. 1974, in Coronal Disturbances, ed. G. A. Newkirk, IAU Symp., 57, 389

Steinolfson, R. S. 1984, Sol. Phys., 94, 193

Temmer, M., Veronig, A. M., Gopalswamy, N., \& Yashiro, S. 2011, Sol. Phys., 273, 421

Thernisien, A., Vourlidas, A., \& Howard, R. A. 2009, Sol. Phys., 256, 111

Torsti, J., Kocharov, L., Teittinen, M., \& Thompson, B. 1999, ApJ, 510, 460

Treumann, R. A., \& LaBelle, J. 1992, ApJ, 399, L167

Vasanth, V., Umapathy, S., Vršnak, B., Žic, T., \& Prakash, O. 2014, Sol. Phys., 289,251

Vršnak, B., Aurass, H., Magdalenić, J., \& Gopalswamy, N. 2001, A\&A, 377, 321

Vršnak, B., Magdalenić, J., Aurass, H., \& Mann, G. 2002, A\&A, 396, 673

Warmuth, A. 2015, Liv. Rev. Sol. Phys., 12, 3

Wibberenz, G., \& Cane, H. V. 2006, ApJ, 650, 1199

Wiedenbeck, M. E., Mason, G. M., Gómez-Herrero, R., et al. 2010, in Twelfth International Solar Wind Conference, AIP Conf. Proc., 1216, 621

Wood, B. E., \& Howard, R. A. 2009, ApJ, 702, 901

Wuelser, J.-P., Lemen, J. R., Tarbell, T. D., et al. 2004, in Telescopes and Instrumentation for Solar Astrophysics, eds. S. Fineschi, \& M. A. Gummin, SPIE Conf. Ser., 5171, 111

Zank, G. P., Rice, W. K. M., \& Wu, C. C. 2000, J. Geophys. Res., 105, 25079

Zimovets, I., Vilmer, N., Chian, A. C.-L., Sharykin, I., \& Struminsky, A. 2012, A\&A, 547, A6

Zucca, P., Carley, E. P., Bloomfield, D. S., \& Gallagher, P. T. 2014a, A\&A, 564, A 47

Zucca, P., Pick, M., Démoulin, P., et al. 2014b, ApJ, 795, 68 


\section{Appendix A: Analysis of interplanetary conditions between 20 and 30 April 2016}

In this section, we evaluate whether the magnetic field line invoked in Sect. 6.3 (Fig. 5), which connects STEREO-B to the western flank of the shock is perturbed by heliospheric structures, such as CMEs and corotating interaction regions (CIRs). To do so, we employ the CME and CIR catalogs produced by the HELCATS $^{1}$ project using images from STEREO.

The catalog uses the fitting technique developed by Rouillard et al. (2010a,b), which is based on J-maps. Through a systematic analysis, Plotnikov et al. (2016) show that all CIRs measured in situ were also detected in white-light imagery between 2007 and 2009.

The range of elongation angles shown in the J-maps of Figs. A.1a and b (vertical axis), goes from $4^{\circ}$ to $74^{\circ}$. This angular range that was imaged by the heliospheric imagers onboard STEREO-A includes the elongation of STEREO-B, shown by the dotted horizontal lines near elongation $70^{\circ}$ in Figs. A.1a and $b$. Hence STEREO-A was at the time imaging plasma flowing between the Sun and STEREO-B (see Fig. A.1c). No large CME propagated outward in the heliospheric images of STEREO-A, other than the event of interest in this paper. The various trajectory estimates listed in the HELCATS Catalogue confirm that the CME studied in this paper propagated to within $20^{\circ}$ of the longitude of STEREO-B at an estimated speed of around $550 \mathrm{~km} \mathrm{~s}^{-1}$. This agrees with the speed of plasma located downstream of the shock, which was measured by STEREO-B at the impact time. We repeated the trajectory analysis by combining the fixed-phi model (Rouillard et al. 2008) with the results of the triangulation work given in Sect. 6.3. The latter gave a longitude of propagation of $200^{\circ}$ along the central axis and a shock passage time at $10 R_{\odot}$ of 16:00 UT on 26 April with a speed of $550 \mathrm{~km} \mathrm{~s}^{-1}$. The time-elongation variation of this hypothetical $\mathrm{CME}$ produces the red track shown in Fig. A.1a. For an average transit speed of $550 \mathrm{~km} \mathrm{~s}^{-1}$, the track very closely matches the leading edge of the CME track that was shown to be the time-varying location of the shock by Wood \& Howard (2009). The derived location of the CME is shown on the view of the ecliptic in Fig. A.1c at 12:00 UT on 27 April 2008.

The J-map in Fig. A.1a also reveals the presence of a pattern of converging tracks that is typical of a CIR passing in the field of view (e.g., Rouillard et al. 2008). Each track corresponds to a density inhomogeneity (or so-called blob) that becomes compressed inside the CIR, acting as a tracer of the CIR progression along a specific longitude. The CIR corotates and density blobs are released periodically from the low corona, which produces this characteristic pattern of tracks in the J-map that acts as a tracer of the longitudinal and radial progression of the CIR (Rouillard et al. 2008). The HELCATS Catalogue confirms the presence of a CIR propagating towards STEREO-B at the time; the CIR pattern extracted from the J-map is shown as the family of black lines overlying the J-map shown in Fig. A.1b. In this calculation, a reference track (red line in Fig. A.1b) is usually assumed to reconstruct the pattern of a converging track. The position of the CIR in the ecliptic plane at 12:00 UT on 27 April 2008, as calculated by the propagation tool, is shown in Fig. A.1d. The CIR is approaching STEREO-B at the time and the tool computed an impact time based on radial propagation and corotation on 29 April 2008 at around 7 UT with a typical uncertainty of $8 \mathrm{~h}$. The interplanetary CME (ICME) shock clearly identified by Wood \& Howard (2009) in whitelight images and tracked in Fig. A.1 a arrives at STEREO-B near 13:00 UT, we conclude that the CME must have encountered the CIR during its radial propagation to STEREO-B.

The complex in situ signature downstream of the shock may result from the complex interaction that must have occurred between the CME and the CIR during the propagation of the CME to STEREO-B. According to Fig. A.1, this complex interaction should have occurred well after 12:00 UT on 27 April 2008. Therefore the magnetic connectivity of STEREO-B to the shock at the time of the early SEP signatures (Sect. 6.3) is not yet affected by that interaction. This is particularly true at the low heights at which the triangulation work was carried out $\left(<15 R_{\odot}\right)$, where CIRs have not yet formed.

1 http://www.helcats-fp7.eu/products.html 
(a) ${ }^{7}$
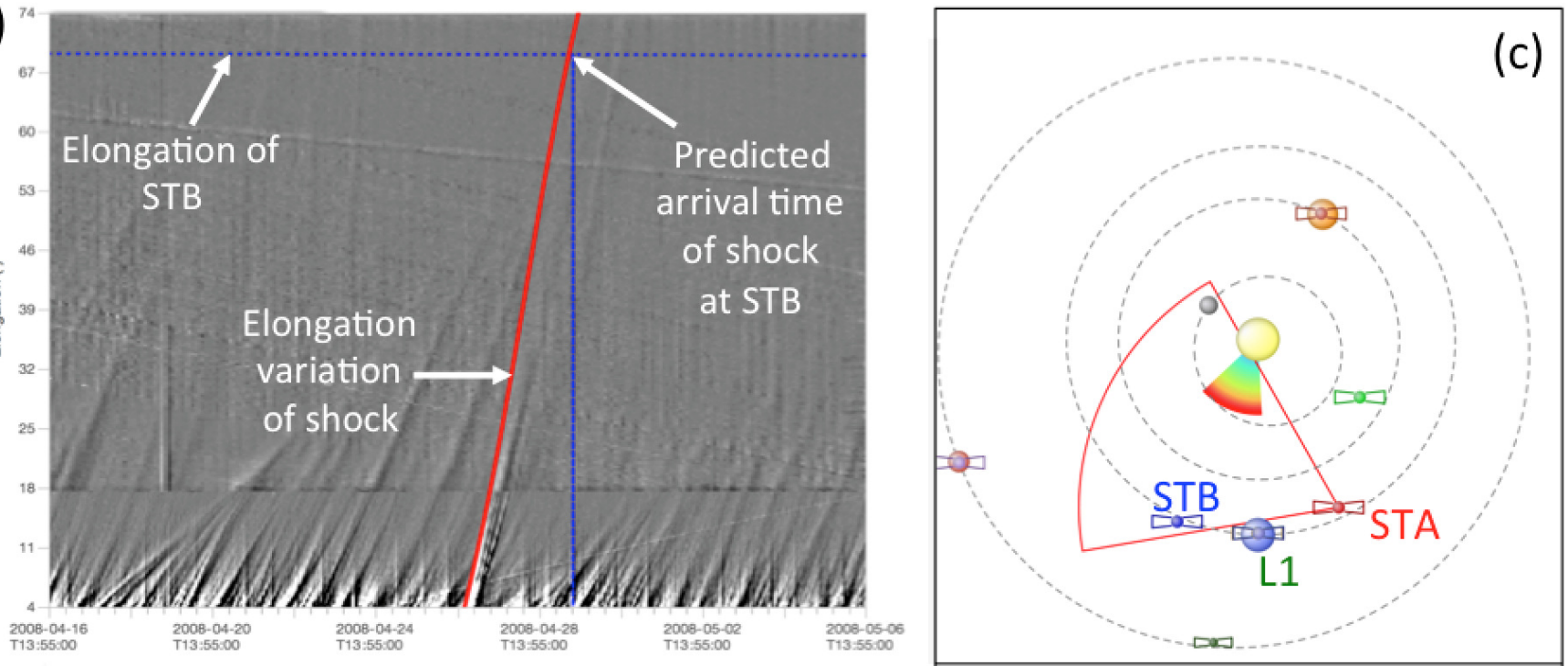

(b)
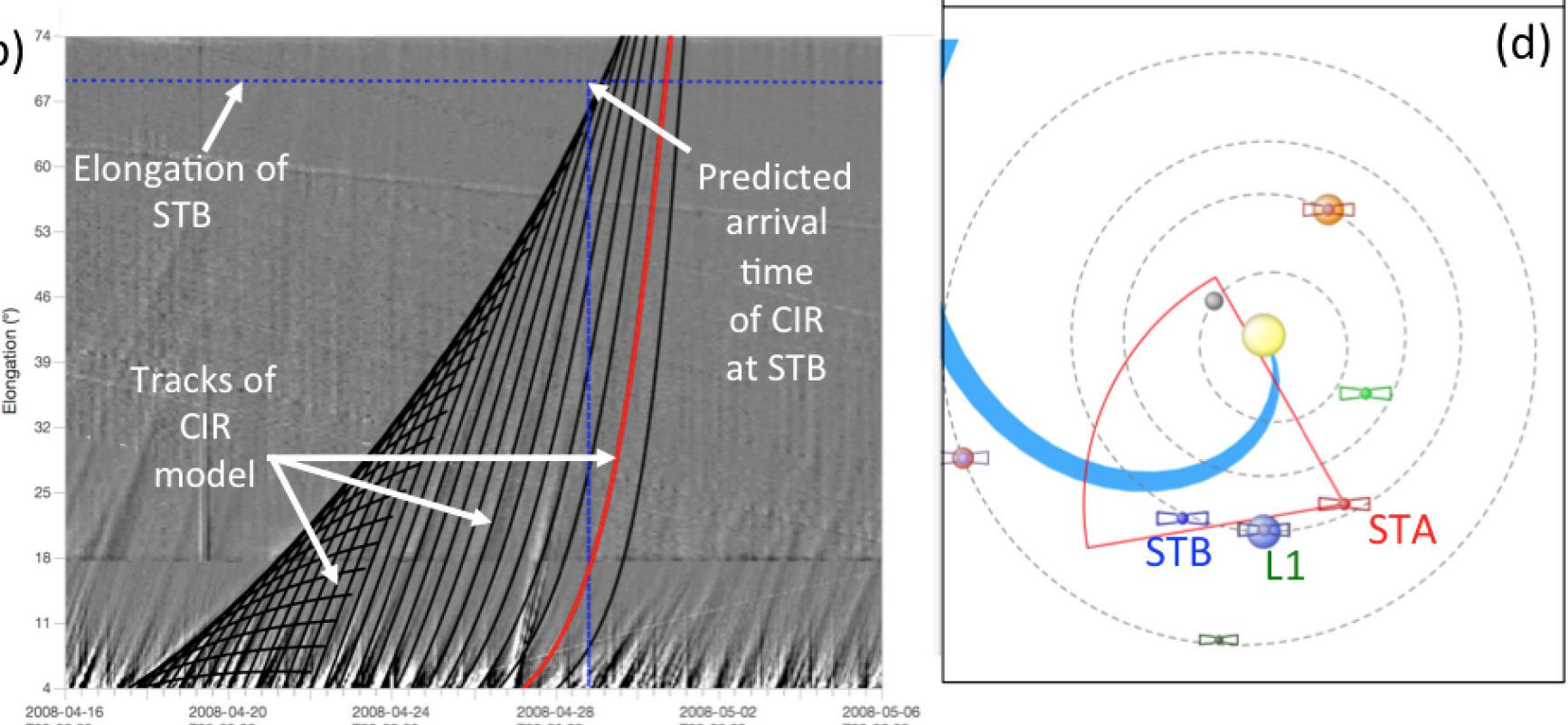
2008-04-16 2008-04-20 2008-04-24 2006-04-26 2006-05-02

Fig. A.1. a), b): J-maps derived from heliospheric imaging made by STEREO-A showing the state of the interplanetary medium between 16 April and 6 May 2008. Each track on these J-maps corresponds to a density structure moving radially outward from the Sun and leaving a strong signature in the white-light images. The horizontal dotted line near the top of the maps shows the elongation of STEREO-B (STB). The inclined red line in a) is the track of a hypothetical CME launched near the time of the 26 April 2008 event and propagating at constant speed $550 \mathrm{~km} \mathrm{~s}^{-1}$. The vertical blue line marks its arrival at STB. c), d): view of the ecliptic plane from solar north with the respective locations of STEREO-A (STA), STB and L1 as well as other planets and probes that are not all labeled for clarity purposes. The angular extents of the J-maps shown in the left-hand columns are shown as red contour lines emanating from the STA. The locations of the CME (c) and CIR (d); blue band) derived by the J-map analysis are shown at 12:00 UT on 27 April 2008. These four panels were produced using the IRAP propagation tool (propagationtool.cdpp. eu). 